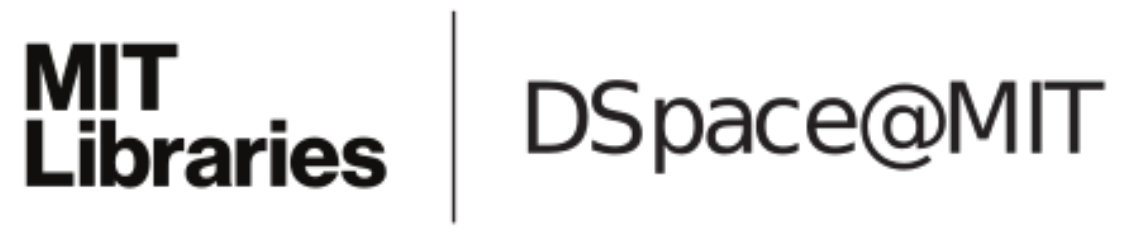

MIT Open Access Articles

A relative Szemerédi theorem

The MIT Faculty has made this article openly available. Please share how this access benefits you. Your story matters.

Citation: Conlon, David, Jacob Fox, and Yufei Zhao. "A Relative Szemerédi Theorem." Geometric and Functional Analysis 25, no. 3 (March 17, 2015): 733-762.

As Published: http://dx.doi.org/10.1007/s00039-015-0324-9

Publisher: Springer Basel

Persistent URL: http://hdl.handle.net/1721.1/106219

Version: Author's final manuscript: final author's manuscript post peer review, without publisher's formatting or copy editing

Terms of use: Creative Commons Attribution-Noncommercial-Share Alike 


\title{
A RELATIVE SZEMERÉDI THEOREM
}

\author{
David Conlon, Jacob Fox And Yufei ZhaO
}

\begin{abstract}
The celebrated Green-Tao theorem states that there are arbitrarily long arithmetic progressions in the primes. One of the main ingredients in their proof is a relative Szemerédi theorem which says that any subset of a pseudorandom set of integers of positive relative density contains long arithmetic progressions. In this paper, we give a simple proof of a strengthening of the relative Szemerédi theorem, showing that a much weaker pseudorandomness condition is sufficient. Our strengthened version can be applied to give the first relative Szemerédi theorem for $k$-term arithmetic progressions in pseudorandom subsets of $\mathbb{Z}_{N}$ of density $N^{-c_{k}}$. The key component in our proof is an extension of the regularity method to sparse pseudorandom hypergraphs, which we believe to be interesting in its own right. From this we derive a relative extension of the hypergraph removal lemma. This is a strengthening of an earlier theorem used by Tao in his proof that the Gaussian primes contain arbitrarily shaped constellations and, by standard arguments, allows us to deduce the relative Szemerédi theorem.
\end{abstract}

\section{Introduction}

The Green-Tao theorem [GT08] states that the primes contain arbitrarily long arithmetic progressions. This result, along with their subsequent work [GT10] on determining the asymptotics for the number of prime $k$-tuples in arithmetic progression, constitutes one of the great breakthroughs in twenty-first century mathematics.

The proof of the Green-Tao theorem has two key steps. The first step, which Green and Tao refer to as the "main new ingredient" of their proof, is to establish a relative Szemerédi theorem. Szemerédi's theorem [Sze75] states that any dense subset of the integers contains arbitrarily long arithmetic progressions. More formally, we have the following theorem, which is stated for $\mathbb{Z}_{N}:=\mathbb{Z} / N \mathbb{Z}$ but easily implies an equivalent statement in the set $[N]:=\{1,2, \ldots, N\}$.

The first author was supported by a Royal Society University Research Fellowship, the second author was supported by a Simons Fellowship, NSF grant DMS-1069197, by an Alfred P. Sloan Fellowship, and by an MIT NEC Corporation Fund Award, and the third author was supported by a Microsoft Research PhD Fellowship. 
Theorem 1.1 (Szemerédi's theorem). For every natural number $k \geq 3$ and every $\delta>0$, as long as $N$ is sufficiently large, any subset of $\mathbb{Z}_{N}$ of density at least $\delta$ contains an arithmetic progression of length $k$.

A relative Szemerédi theorem is a similar statement where the ground set is no longer the set $\mathbb{Z}_{N}$ but rather a sparse pseudorandom subset of $\mathbb{Z}_{N}$.

The second step in their proof is to show that the primes are a dense subset of a pseudorandom set of "almost primes", sufficiently pseudorandom that the relative Szemerédi theorem holds. Then, since the primes are a dense subset of this pseudorandom set, an application of the relative Szemerédi theorem implies that the primes contain arbitrarily long arithmetic progressions. This part of the proof uses some ideas from the work of Goldston and Yıldırım [GY03] (and was subsequently simplified in [Tao]).

In the work of Green and Tao, the pseudorandomness conditions on the ground set are known as the linear forms condition and the correlation condition. Roughly speaking, both of these conditions say that, in terms of the number of solutions to certain linear systems of equations, the set behaves like a random set of the same density. A natural question is whether these pseudorandomness conditions can be weakened. We address this question by giving a simple proof for a strengthening of the relative Szemerédi theorem, showing that a weak linear forms condition is sufficient for the theorem to hold.

This improvement has two aspects. We remove the correlation condition entirely but we also reduce the set of linear forms for which the correct count is needed. In particular, we remove those corresponding to the dual function condition, a pointwise boundedness condition stated explicitly by Tao [Tao06a] in his work on constellations in the Gaussian primes but also used implicitly in [GT08].

To state the main theorem, we will assume the definition of the $k$-linear forms condition. The formal definition, which may be found in Section 2 below, is stated for measures rather than sets but we will ignore this relatively minor distinction here, reserving a more complete discussion of our terminology for there.

Theorem 1.2 (Relative Szemerédi theorem). For every natural number $k \geq 3$ and every $\delta>0$, if $S \subset \mathbb{Z}_{N}$ satisfies the $k$-linear forms condition and $N$ is sufficiently large, then any subset of $S$ of relative density at least $\delta$ contains an arithmetic progression of length $k$.

One of the immediate advantages of this theorem is that it simplifies the proof of the Green-Tao theorem. In addition to giving a simple proof of the relative Szemerédi theorem, it removes the need for the number-theoretic estimates involved in establishing the correlation condition for the almost primes. A further advantage is that, by removing the correlation condition, the relative Szemerédi theorem now applies to pseudorandom subsets of $\mathbb{Z}_{N}$ of density $N^{-c_{k}}$. With the correlation condition, one could only hope for such a theorem down to densities of the form $N^{-o(1)}$.

While the relative Szemerédi theorem is the main result of this paper, the main advance is an approach to regularity in sparse pseudorandom hypergraphs. This 
allows us to prove analogues of several well-known combinatorial theorems relative to sparse pseudorandom hypergraphs. In particular, we prove a sparse analogue of the hypergraph removal lemma. It is from this that we derive our relative Szemerédi theorem. As always, applying the regularity method has two steps, a regularity lemma and a counting lemma. We provide novel approaches to both.

A counting lemma for subgraphs of sparse pseudorandom graphs was already proved by the authors in [CFZ14]. In this paper, we simplify and streamline the approach taken there in order to prove a counting lemma for subgraphs of sparse pseudorandom hypergraphs. This result is the key technical step in our proof and, perhaps, the main contribution of this paper. Apart from the obvious difficulties in passing from graphs to hypergraphs, the crucial difference between this paper and [CFZ14] is in the type of pseudorandomness considered. For graphs, we have a long-established notion of pseudorandomness known as jumbledness. The greater part of [CFZ14] is then concerned with optimizing the jumbledness condition which is necessary for counting a particular graph $H$. For hypergraphs, we use an analogue of the linear forms condition first considered by Tao [Tao06a]. This says that our hypergraph is pseudorandom enough for counting $H$ within subgraphs if it contains asymptotically the correct count for the 2-blow-up of $H$ and all its subgraphs.

We also use an alternative approach to regularity in sparse hypergraphs. While it would be natural to use a sparse hypergraph regularity lemma (and, following our approach in [CFZ14], this was how we initially proceeded), it suffices to use a weak sparse hypergraph regularity lemma which is an extension of the weak regularity lemma of Frieze and Kannan [FK99]. This is also closely related to the transference theorem used by Green and Tao (see, for example, [Gow10] or [RTTV08, TTV09], where it is also referred to as the dense model theorem).

With both a regularity lemma and a counting lemma in place, it is then a straightforward matter to prove a relative extension of the famous hypergraph removal lemma [Gow07, NRS06, RS04, RS06, Tao06b]. Such a theorem was first derived by Tao [Tao06a] in his work on constellations in the Gaussian primes but, like the Green-Tao relative Szemerédi theorem, needs both a correlation condition and a dual function condition. ${ }^{1}$ Our approach removes these conditions. The final step in the proof of the relative Szemerédi theorem is then a standard reduction used to derive Szemerédi's theorem from the hypergraph removal lemma. The details of this reduction already appear in [Tao06a] but we include them here for completeness. In fact, the paper is self-contained apart from assuming the hypergraph removal lemma.

In Section 2, we state our results, including the relative Szemerédi theorem and the removal, regularity, and counting lemmas. In Section 3, we deduce the relative multidimensional Szemerédi theorem from our relative hypergraph removal lemma. In Section 4, we prove the removal lemma from the regularity and counting lemmas. We will prove our weak sparse hypergraph regularity lemma in Section 5 and

\footnotetext{
${ }^{1}$ The problem of relative hypergraph removal was also recently considered by Towsner [Tow].
} 
the associated counting lemma in Section 6. We conclude, in Section 7, with some remarks.

\section{Definitions and Results}

Notation. Dependence on $N$. We consider functions $\nu=\nu^{(N)}$, where $N$ (usually suppressed) is assumed to be some large integer. We write $o(1)$ for a quantity that tends to zero as $N \rightarrow \infty$.

Expectation. We write $\mathbb{E}\left[f\left(x_{1}, x_{2}, \ldots\right) \mid x_{1} \in A_{1}, x_{2} \in A_{2}, \ldots\right]$ for the expectation of $f\left(x_{1}, x_{2}, \ldots\right)$ when each $x_{i}$ is chosen uniformly and independently at random from $A_{i}$.

2.1 A relative Szemerédi theorem. Here is an equivalent weighted version of Szemerédi's theorem as formulated, for example, in [GT08, Prop. 2.3].

Theorem 2.1 (Szemerédi's theorem, weighted version). For every $k \geq 3$ and $\delta>$ 0 , there exists $c>0$ such that for $N$ sufficiently large and any nonnegative function $f: \mathbb{Z}_{N} \rightarrow[0,1]$ satisfying $\mathbb{E}[f] \geq \delta$,

$$
\mathbb{E}\left[f(x) f(x+d) f(x+2 d) \cdots f(x+(k-1) d) \mid x, d \in \mathbb{Z}_{N}\right] \geq c .
$$

A relative Szemerédi theorem would instead ask for the nonnegative function $f$ to be bounded above by a measure $\nu$ instead of the constant function $f$. For us, a measure will be any nonnegative function on $\mathbb{Z}_{N}$. We do not explicitly assume the additional condition that

$$
\mathbb{E}\left[\nu(x) \mid x \in \mathbb{Z}_{N}\right]=1+o(1),
$$

but this property follows from the linear forms condition that we will now assume. Such measures are more general than subsets, as any subset $S \subseteq \mathbb{Z}_{N}$ (e.g., in Theorem 1.2) can be thought of as a measure on $\mathbb{Z}_{N}$ taking value $N /|S|$ on $S$ and 0 elsewhere. The dense case, as in Theorem 2.1, corresponds to taking $\nu=1$. Our notion of pseudorandomness for measures $\nu$ on $\mathbb{Z}_{N}$ is now as follows.

Definition 2.2 (Linear forms condition). A nonnegative function $\nu=\nu^{(N)}: \mathbb{Z}_{N} \rightarrow$ $\mathbb{R}_{\geq 0}$ is said to obey the $k$-linear forms condition if one has

$\mathbb{E}\left[\prod_{j=1}^{k} \prod_{\omega \in\{0,1\}^{[k] \backslash\{j\}}} \nu\left(\sum_{i=1}^{k}(i-j) x_{i}^{\left(\omega_{i}\right)}\right)^{n_{j, \omega}} \mid x_{1}^{(0)}, x_{1}^{(1)}, \ldots, x_{k}^{(0)}, x_{k}^{(1)} \in \mathbb{Z}_{N}\right]=1+o(1)$

for any choices of exponents $n_{j, \omega} \in\{0,1\}$.

EXAmPLE 2.3. For $k=3$, condition (2) says that

$$
\begin{gathered}
\mathbb{E}\left[\nu(y+2 z) \nu\left(y^{\prime}+2 z\right) \nu\left(y+2 z^{\prime}\right) \nu\left(y^{\prime}+2 z^{\prime}\right) \nu(-x+z) \nu\left(-x^{\prime}+z\right) \nu\left(-x+z^{\prime}\right) \nu\left(-x^{\prime}+z^{\prime}\right)\right. \\
\left.\cdot \nu(-2 x-y) \nu\left(-2 x^{\prime}-y\right) \nu\left(-2 x-y^{\prime}\right) \nu\left(-2 x^{\prime}-y^{\prime}\right) \mid x, x^{\prime}, y, y^{\prime}, z, z^{\prime} \in \mathbb{Z}_{N}\right]=1+o(1)
\end{gathered}
$$


and similar conditions hold if one or more of the twelve $\nu$ factors in the expectation are erased.

Our linear forms condition is much weaker than that used in Green and Tao [GT08]. In particular, Green and Tao need to assume that pointwise estimates such as

$$
\mathbb{E}\left[\nu(a+x) \nu(a+y) \nu(a+x+y) \mid x, y \in \mathbb{Z}_{N}\right]=1+o(1)
$$

hold uniformly over all $a \in \mathbb{Z}_{N}$. Such linear forms do not arise in our proof. Moreover, to prove their relative Szemerédi theorem, Green and Tao need to assume a further pseudorandomness condition, which they call the correlation condition. This condition also does not arise in our proofs. Indeed, we prove that a relative Szemerédi theorem holds given only the linear forms condition defined above.

Theorem 2.4 (Relative Szemerédi theorem). For every $k \geq 3$ and $\delta>0$, there exists $c>0$ such that if $\nu: \mathbb{Z}_{N} \rightarrow \mathbb{R}_{\geq 0}$ satisfies the $k$-linear forms condition, $N$ is sufficiently large, and $f: \mathbb{Z}_{N} \rightarrow \mathbb{R}_{\geq 0}$ satisfies $0 \leq f(x) \leq \nu(x)$ for all $x \in \mathbb{Z}_{N}$ and $\mathbb{E}[f] \geq \delta$, then

$$
\mathbb{E}\left[f(x) f(x+d) f(x+2 d) \cdots f(x+(k-1) d) \mid x, d \in \mathbb{Z}_{N}\right] \geq c .
$$

We note that both here and in Theorem 1.2 , the phrase " $N$ is sufficiently large" indicates not only a dependency on $\delta$ and $k$ as in the usual version of Szemerédi's theorem but also a dependency on the $o(1)$ term in the linear forms condition. We will make a similar assumption in many of the theorems stated below.

We prove Theorem 2.4 using a new relative hypergraph removal lemma. ${ }^{2}$ In the next subsection, we set up the notation for hypergraphs and state the corresponding pseudorandomness hypothesis.

2.2 Hypergraphs. We borrow most of our notation and definitions from Tao [Tao06a, Tao06b].

Definition 2.5 (Hypergraphs). Let $J$ be a finite set and $r$ a positive integer. Define $\left(\begin{array}{l}J \\ r\end{array}\right)=\{e \subseteq J:|e|=r\}$ to be the set of all $r$-element subsets of $J$. An $r$-uniform hypergraph on $J$ is defined to be any subset $H \subseteq\left(\begin{array}{l}J \\ r\end{array}\right)$.

Definition 2.6 (Hypergraph system). A hypergraph system is a quadruple $V=$ $\left(J,\left(V_{j}\right)_{j \in J}, r, H\right)$, where $J$ is a finite set, $\left(V_{j}\right)_{j \in J}$ is a collection of finite non-empty sets indexed by $J, r$ is a positive integer, and $H \subseteq\left(\begin{array}{l}J \\ r\end{array}\right)$ is an r-uniform hypergraph. For any $e \subseteq J$, we set $V_{e}:=\prod_{j \in e} V_{j}$. For any $x=\left(x_{j}\right)_{j \in J} \in V_{J}$ and any subset $J^{\prime} \subseteq J$, we write $x_{J^{\prime}}=\left(x_{j}\right)_{j \in J^{\prime}} \in V_{J^{\prime}}$ to mean the natural projection of $x$ onto the

${ }^{2}$ Green and Tao [GT08] prove a transference result that allows them to apply the dense version of Szemerédi's theorem as a black box. This allows them to show that the optimal $c$ in (3) can be taken to be the same as the optimal $c$ in (1). The proof in this paper goes through the hypergraph removal lemma and thus does not obtain the same $c$. Nevertheless, one can obtain our result with the same $c$ by modifying the argument to an arithmetic setting, as done by the third author in a follow-up paper [Zh14]. 
coordinates $J^{\prime}$. Finally, for any $e \subseteq J$, we write $\partial e$ for the set $\{f \subsetneq e:|f|=|e|-1\}$, the skeleton of $e$.

Definition 2.7 (Weighted hypergraphs). Let $V=\left(J,\left(V_{j}\right)_{j \in J}, r, H\right)$ be a hypergraph system. A weighted hypergraph on $V$ is a collection $g=\left(g_{e}\right)_{e \in H}$ of functions $g_{e}: V_{e} \rightarrow \mathbb{R}_{\geq 0}$ indexed by $H$. We write 0 and 1 to denote the constant-valued weighted hypergraphs of uniform weight 0 and 1, respectively. Given two weighted hypergraphs $g$ and $\nu$ on the same hypergraph system, we write $g \leq \nu$ to mean that $g_{e} \leq \nu_{e}$ for all $e$, which in turn means that $g_{e}\left(x_{e}\right) \leq \nu_{e}\left(x_{e}\right)$ for all $x_{e} \in V_{e}$.

The weighted hypergraph $\nu$ plays an analogous role to the $\nu$ in Theorem 2.4 , with $\nu=1$ again corresponding to the dense case. We have an analogous linear forms condition for $\nu$ as a weighted hypergraph. We use the following indexing notation. For a finite set $e$ and $\omega \in\{0,1\}^{e}$, we write $x_{e}^{(\omega)}$ to mean the tuple $\left(x_{j}^{\left(\omega_{j}\right)}\right)_{j \in e}$. We also write $x_{e}^{(0)}:=\left(x_{j}^{(0)}\right)_{j \in e}$ and similarly with $x_{e}^{(1)}$.

Definition 2.8 (Linear forms condition). A weighted hypergraph $\nu=\nu^{(N)}$ on the hypergraph system $V=V^{(N)}=\left(J,\left(V_{j}^{(N)}\right)_{j \in J}, r, H\right)$ is said to obey the $H$-linear forms condition (or simply the linear forms condition if there is no confusion) if one has

$$
\mathbb{E}\left[\prod_{e \in H} \prod_{\omega \in\{0,1\}^{e}} \nu_{e}\left(x_{e}^{(\omega)}\right)^{n_{e, \omega}} \mid x_{J}^{(0)}, x_{J}^{(1)} \in V_{J}\right]=1+o(1)
$$

for any choices of exponents $n_{e, \omega} \in\{0,1\}$.

ExAmple 2.9. Let $H$ be the set of all pairs in $J=\{1,2,3\}$. The linear forms condition says that

$$
\begin{gathered}
\mathbb{E}\left[\prod_{i j=12,13,23} \nu_{i j}\left(x_{i}, x_{j}\right) \nu_{i j}\left(x_{i}^{\prime}, x_{j}\right) \nu_{i j}\left(x_{i}, x_{j}^{\prime}\right) \nu_{i j}\left(x_{i}^{\prime}, x_{j}^{\prime}\right) \mid x_{1}, x_{1}^{\prime} \in V_{1},\right. \\
\left.x_{2}, x_{2}^{\prime} \in V_{2}, x_{3}, x_{3}^{\prime} \in V_{3}\right]=1+o(1)
\end{gathered}
$$

and similarly if one or more of the twelve $\nu$ factors are deleted. This expression represents the weighted homomorphism density of $K_{2,2,2}$ in the weighted tripartite graph given by $\nu$, as illustrated in Figure $1 \mathrm{~b}$ (the vertices of $K_{2,2,2}$ must map into the corresponding parts). Deleting some $\nu$ factors corresponds to considering various subgraphs of $K_{2,2,2}$, e.g., Figure 1c.

In general, the $H$-linear forms condition says that $\nu$ has roughly the expected density for the 2-blow-up ${ }^{3}$ of $H$ as well as any subgraph of the 2-blow-up. Our

\footnotetext{
3 By the 2-blow-up of $H$ we mean the hypergraph consisting of vertices $j^{(0)}, j^{(1)}$ for each $j \in J$, and edges $e^{(\omega)}:=\left\{j^{\left(\omega_{j}\right)}: j \in e\right\}$ for any $e \in H$ and $\omega \in\{0,1\}^{e}$. We actually do not need the
} 


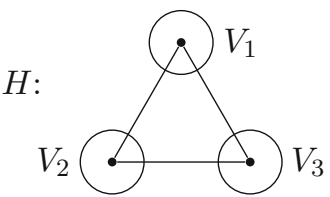

(a)

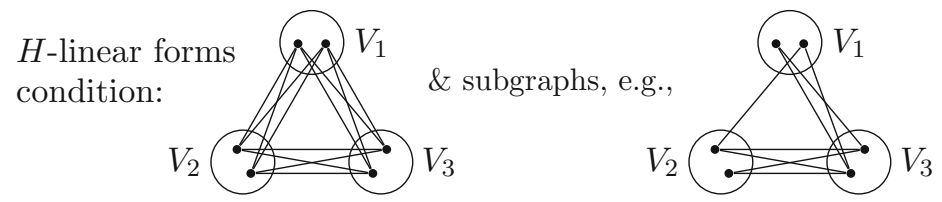

(b)

(c)

Figure 1: Linear forms conditions for $H=K_{3}$. See Example 2.9

linear forms condition for hypergraphs coincides with the one used by Tao [Tao06a, Def. 2.8], although in [Tao06a] one assumes additional pseudorandomness hypotheses on $\nu$ known as the dual function condition and the correlation condition.

2.3 Hypergraph removal lemma. The hypergraph removal lemma was first proved by Gowers [Gow07] and by Nagle et al. [NRS06, RS04, RS06]. It states that for every $r$-uniform hypergraph $H$ on $h$ vertices, every $r$-uniform hypergraph on $n$ vertices with $o\left(n^{h}\right)$ copies of $H$ can be made $H$-free by removing $o\left(n^{r}\right)$ edges. As first explicitly stated and proved by Tao [Tao06b], the proof of the hypergraph removal lemma further gives that the edges can be removed in a low complexity way (this idea will soon be made formal). We will use a slightly stronger version, where edges are given weights in the interval $[0,1]$. This readily follows from the usual version by a simple rounding argument, as done in [Tao06a, Thm. 3.7]. We state this result as Theorem 2.11 below.

Definition 2.10. For any set $e$ of size $r$ and any $E_{e} \subseteq V_{e}=\prod_{j \in e} V_{j}$, we define the complexity of $E_{e}$ to be the minimum integer $T$ such that there is a partition of $E_{e}$ into $T$ sets $E_{e, 1}, \ldots, E_{e, T}$ so that each $E_{e, i}$ is the set of $r$-cliques of some $(r-1)$ uniform hypergraph, meaning that there exists some $B_{f, i} \subseteq V_{f}$ for each $f \in \partial e$ so that $1_{E_{e, i}}\left(x_{e}\right)=\prod_{f \in \partial e} 1_{B_{f, i}}\left(x_{f}\right)$ for all $x_{e} \in V_{e}$.

Theorem 2.11 (Weighted hypergraph removal lemma). For every $\epsilon>0$ and finite set $J$, there exists $\delta>0$ and $T>0$ such that the following holds. Let $V=\left(J,\left(V_{j}\right)_{j \in J}, r, H\right)$ be a hypergraph system. Let $g$ be a weighted hypergraph on $V$ satisfying $0 \leq g \leq 1$ and

$$
\mathbb{E}\left[\prod_{e \in H} g_{e}\left(x_{e}\right) \mid x \in V_{J}\right] \leq \delta .
$$

Footnote 3 continued

full strength of this assumption. It suffices to assume that $\nu$ has roughly the expected density for any subgraph of a weak 2-blow-up of $H$, where by a weak 2-blow-up we mean the following. Fix some edge $e_{1} \in H$ (we will need to assume the condition for all $e_{1}$ ). The weak 2-blow-up of $H$ with respect to $e_{1}$ is the subgraph of the usual 2-blow-up consisting of all edges $e^{(\omega)}$ where $\omega_{i}=\omega_{j}$ for any $i, j \in e \backslash e_{1}$. This weaker version of the $H$-linear forms condition is all we shall use for the proof, although everything to follow will be stated as in Definition 2.8 for clarity. 
Then for each $e \in H$ there exists a set $E_{e}^{\prime} \subseteq V_{e}$ for which $V_{e} \backslash E_{e}^{\prime}$ has complexity at most $T$ and such that

$$
\prod_{e \in H} 1_{E_{e}^{\prime}}\left(x_{e}\right)=0 \text { for all } x \in V_{J}
$$

and for all $e \in H$ one has

$$
\mathbb{E}\left[g_{e}\left(x_{e}\right) 1_{V_{e} \backslash E_{e}^{\prime}}\left(x_{e}\right) \mid x_{e} \in V_{e}\right] \leq \epsilon .
$$

We prove a relativized extension of the hypergraph removal lemma. A relative hypergraph removal lemma was already established by Tao in [Tao06a], where he assumed the majorizing measure satisfies three conditions: the linear forms condition, the correlation condition, and the dual function condition. We again show that a linear forms condition is sufficient.

Theorem 2.12 (Relative hypergraph removal lemma). For every $\epsilon>0$ and finite set $J$, there exists $\delta>0$ and $T>0$ such that the following holds. Let $V=\left(J,\left(V_{j}\right)_{j \in J}, r, H\right)$ be a hypergraph system. Let $\nu$ and $g$ be weighted hypergraphs on $V$. Suppose $0 \leq g \leq \nu, \nu$ satisfies the $H$-linear forms condition, and $N$ is sufficiently large. If

$$
\mathbb{E}\left[\prod_{e \in H} g_{e}\left(x_{e}\right) \mid x \in V_{J}\right] \leq \delta,
$$

then for each $e \in H$ there exists a set $E_{e}^{\prime} \subseteq V_{e}$ for which $V_{e} \backslash E_{e}^{\prime}$ has complexity at most $T$ and such that

$$
\prod_{e \in H} 1_{E_{e}^{\prime}}\left(x_{e}\right)=0 \quad \text { for all } x \in V_{J}
$$

and for all $e \in H$ one has

$$
\mathbb{E}\left[g_{e}\left(x_{e}\right) 1_{V_{e} \backslash E_{e}^{\prime}}\left(x_{e}\right) \mid x_{e} \in V_{e}\right] \leq \epsilon .
$$

In Section 4 we will deduce Theorem 2.12 from Theorem 2.11 by applying the weak regularity lemma and the counting lemma which are stated in the next two subsections.

2.4 Weak hypergraph regularity. The Frieze-Kannan weak regularity lemma [FK99] allows one to approximate in cut-norm a matrix (or graph) with entries in the interval $[0,1]$ by another matrix of low complexity. A major advantage over simply applying Szemerédi's regularity lemma is that the complexity has only an exponential dependence on the approximation parameter, as opposed to the tower-type bound that is incurred by Szemerédi's regularity lemma. Unfortunately, these regularity lemmas are not meaningful for sparse graphs as the error term is too large in this setting. Following sparse extensions of Szemerédi's regularity lemma by Kohayakawa [Koh97] and Rödl, a sparse extension of the weak regularity lemma was proved by Bollobás and Riordan [BR09] and by Coja-Oghlan 
et al. [CCF09]. In [CCF09], they further generalize this to $r$-dimensional tensors (or $r$-uniform hypergraphs), but it only gives an approximation which is close in density on all hypergraphs induced by large vertex subsets. In order to prove a relative hypergraph removal lemma, we will need a stronger approximation, which is close in density on all dense $r$-uniform hypergraphs formed by the clique set of some $(r-1)$-uniform hypergraph. In Section 5 , we will prove a more general sparse regularity lemma. For now, we state the result in the form that we need.

The weak regularity lemma approximates a weighted hypergraph $g$ on $V$ by another weighted hypergraph $\tilde{g}$ of bounded complexity which satisfies $0 \leq \tilde{g} \leq 1$. One can think of $\tilde{g}$ as a dense approximation of $g$. The following definition makes precise in what sense $\tilde{g}$ approximates $g$.

Definition 2.13 (Discrepancy pair). Let $e$ be a finite set and $g_{e}, \tilde{g}_{e}: V_{e} \rightarrow \mathbb{R}_{\geq 0}$ be two nonnegative functions. We say that $\left(g_{e}, \tilde{g}_{e}\right)$ is an $\epsilon$-discrepancy pair if for all subsets $B_{f} \subseteq V_{f}, f \in \partial e$, one has

$$
\left|\mathbb{E}\left[\left(g_{e}\left(x_{e}\right)-\tilde{g}_{e}\left(x_{e}\right)\right) \prod_{f \in \partial e} 1_{B_{f}}\left(x_{f}\right) \mid x_{e} \in V_{e}\right]\right| \leq \epsilon .
$$

For two weighted hypergraphs $g$ and $\tilde{g}$ on $\left(J,\left(V_{j}\right)_{j \in J}, r, H\right)$, we say that $(g, \tilde{g})$ is an $\epsilon$-discrepancy pair if $\left(g_{e}, \tilde{g}_{e}\right)$ is an $\epsilon$-discrepancy pair for all $e \in H$.

One needs an additional hypothesis on $g$ in order to prove a weak regularity lemma. The condition roughly says that $g$ contains "no dense spots."

Definition 2.14 (Upper regular). Let e be a finite set, $g_{e}: V_{e} \rightarrow \mathbb{R}_{\geq 0}$ a nonnegative function, and $\eta>0$. We say that $g_{e}$ is upper $\eta$-regular if for all subsets $B_{f} \subseteq V_{f}$, $f \in \partial e$, one has

$$
\mathbb{E}\left[\left(g_{e}\left(x_{e}\right)-1\right) \prod_{f \in \partial e} 1_{B_{f}}\left(x_{f}\right) \mid x_{e} \in V_{e}\right] \leq \eta .
$$

A hypergraph $g$ on on $\left(J,\left(V_{j}\right)_{j \in J}, r, H\right)$ is upper $\eta$-regular if $g_{e}$ is upper $\eta$-regular for all $e \in H$.

Note that unlike (5), there is no absolute value on the left-hand side of (6). The upper regularity hypothesis is needed for establishing the sparse regularity lemma. Fortunately, this mild hypothesis is automatically satisfied in our setting. We will say more about this in Section 6.2.

Lemma 2.15. Let $V=\left(J,\left(V_{j}\right)_{j \in J}, r, H\right)$ be a hypergraph system. Let $\nu$ and $g$ be weighted hypergraphs on $V$. Suppose $0 \leq g \leq \nu$ and $\nu$ satisfies the $H$-linear forms condition. Then $g$ is upper o(1)-regular.

Define the complexity of a function $g: V_{e} \rightarrow[0,1]$ to be the minimum $T$ such that there is a partition of $V_{e}$ into $T$ subgraphs $S_{1}, \ldots, S_{T}$, each of which is the set of 
$r$-cliques of some $(r-1)$-uniform hypergraph (see Definition 2.10), and such that $g$ is constant on each $S_{i}$. We state the regularity lemma below with a complexity bound on $\tilde{g}$, although the complexity bound will not actually be needed for our application.

Theorem 2.16 (Sparse weak regularity lemma). For any $\epsilon>0$ and function $g: V_{1} \times \cdots \times V_{r} \rightarrow \mathbb{R}_{\geq 0}$ which is upper $\eta$-regular with $\eta \leq 2^{-40 r / \epsilon^{2}}$, there exists $\tilde{g}: V_{1} \times \cdots \times V_{r} \rightarrow[0,1]$ with complexity at most $2^{20 r / \epsilon^{2}}$ such that $(g, \tilde{g})$ is an $\epsilon$-discrepancy pair.

The special case $r=2$ is the sparse extension of the Frieze-Kannan weak regularity lemma.

2.5 Counting lemma. Informally, the counting lemma says that if $(g, \tilde{g})$ is an $\epsilon$-discrepancy pair, with the additional assumption that $g \leq \nu$ and $\tilde{g} \leq 1$, then the density of $H$ in $\tilde{g}$ is close to the density of $H$ in $g$. This sparse counting lemma is perhaps the most novel ingredient in this paper.

Theorem 2.17 (Counting lemma). For every $\gamma>0$ and finite set $J$, there exists an $\epsilon>0$ so that the following holds. Let $V=\left(J,\left(V_{j}\right)_{j \in J}, r, H\right)$ be a hypergraph system and $\nu, g, \tilde{g}$ be weighted hypergraphs on $V$. Suppose that $\nu$ satisfies the $H$ linear forms condition and $N$ is sufficiently large. Suppose also that $0 \leq g \leq \nu$, $0 \leq \tilde{g} \leq 1$, and $(g, \tilde{g})$ is an $\epsilon$-discrepancy pair. Then

$$
\left|\mathbb{E}\left[\prod_{e \in H} g_{e}\left(x_{e}\right) \mid x \in V_{J}\right]-\mathbb{E}\left[\prod_{e \in H} \tilde{g}_{e}\left(x_{e}\right) \mid x \in V_{J}\right]\right| \leq \gamma .
$$

As a corollary, Theorem 2.17 also holds if the hypothesis $0 \leq \tilde{g} \leq 1$ is replaced by $0 \leq \tilde{g} \leq \nu$. Indeed, we can use the weak regularity lemma, Theorem 2.16, to find a common 1-bounded approximation to $g$ and $\tilde{g}$. The result then follows from Theorem 2.17 and the triangle inequality.

To summarize, to get a counting lemma for a fixed hypergraph $H$ in a subgraph of a pseudorandom host hypergraph, it suffices to know that the host hypergraph has approximately the expected count for a somewhat larger family of hypergraphs (namely, subgraphs of the 2-blow-up of $H$ ).

\section{The Relative Szemerédi Theorem}

In this section, we deduce the relative Szemerédi theorem, Theorem 2.4, from the relative hypergraph removal lemma, Theorem 2.12. We use the relative hypergraph removal lemma to prove a relative arithmetic removal lemma, Theorem 3.3. This result then easily implies a relative version of the multidimensional Szemerédi theorem of Furstenberg and Katznelson [FK78]. This is Theorem 3.1 below. The relative Szemerédi theorem, Theorem 2.4, follows as a special case of Theorem 3.1 by setting 
$Z=Z^{\prime}=\mathbb{Z}_{N}$ and $\phi_{j}(d)=(j-1) d$. One may easily check that the linear forms condition for the resulting hypergraph is satisfied if $\nu: \mathbb{Z}_{N} \rightarrow \mathbb{R}_{\geq 0}$ satisfies the $k$-linear forms condition.

The statement and proof of Theorem 3.1 closely follows the write-up in Tao [Tao06a, Thm 2.18], adapted in a straightforward way to our new pseudorandomness conditions as well as to the slightly more general setting of functions instead of subsets. Earlier versions of this type of argument for deducing Szemerédi-type results (in the dense setting) from graph and hypergraph removal lemmas were given by Ruzsa and Szemerédi [RS78], Frankl and Rödl [FR02], and Solymosi [Sol03,Sol04].

Theorem 3.1 (Relative multidimensional Szemerédi theorem). For a finite set $J$ and $\delta>0$, there exists $c>0$ so that the following holds. Let $Z, Z^{\prime}$ be two finite additive groups and let $\left(\phi_{j}\right)_{j \in J}$ be a finite collection of group homomorphisms $\phi_{j}$ : $Z \rightarrow Z^{\prime}$ from $Z$ to $Z^{\prime}$. Assume that the elements $\left\{\phi_{i}(d)-\phi_{j}(d): i, j \in J, d \in Z\right\}$ generate $Z^{\prime}$ as an abelian group. Let $\nu: Z^{\prime} \rightarrow \mathbb{R}_{\geq 0}$ be a nonnegative function with the property that in the hypergraph system $V=\left(J,\left(V_{j}\right)_{j \in J}, r, H\right)$, with $V_{j}:=Z$, $r:=|J|-1$, and $H:=\left(\begin{array}{l}J \\ r\end{array}\right)$, the weighted hypergraph $\left(\nu_{e}\right)_{e \in H}$ defined by

$$
\nu_{J \backslash\{j\}}\left(\left(x_{i}\right)_{i \in J \backslash\{j\}}\right):=\nu\left(\sum_{i \in J \backslash\{j\}}\left(\phi_{i}\left(x_{i}\right)-\phi_{j}\left(x_{i}\right)\right)\right)
$$

satisfies the $H$-linear forms condition. Assume that $N$ is sufficiently large. Then, for any $f: Z^{\prime} \rightarrow \mathbb{R}_{\geq 0}$ satisfying $0 \leq f(x) \leq \nu(x)$ for all $x \in Z^{\prime}$ and $\mathbb{E}[f] \geq \delta$,

$$
\mathbb{E}\left[\prod_{j \in J} f\left(a+\phi_{j}(d)\right) \mid a \in Z^{\prime}, d \in Z\right] \geq c .
$$

Example 3.2. Let $S \subset \mathbb{Z}_{N} \times \mathbb{Z}_{N}$. Suppose the associated measure $\nu=\frac{N}{|S|} 1_{S}$ satisfies

$$
\begin{aligned}
& \mathbb{E}\left[\nu(x, y) \nu\left(x^{\prime}, y\right) \nu\left(x, y^{\prime}\right) \nu\left(x^{\prime}, y^{\prime}\right) \nu(x, z-x) \nu\left(x^{\prime}, z-x^{\prime}\right) \nu\left(x, z^{\prime}-x\right) \nu\left(x^{\prime}, z^{\prime}-x^{\prime}\right)\right. \\
& \left.\cdot \nu(z-y, y) \nu\left(z-y^{\prime}, y^{\prime}\right) \nu\left(z^{\prime}-y, y\right) \nu\left(z^{\prime}-y^{\prime}, y^{\prime}\right) \mid x, x^{\prime}, y, y^{\prime}, z, z^{\prime} \in \mathbb{Z}_{N}\right]=1+o(1)
\end{aligned}
$$

and similar conditions hold if any subset of the twelve $\nu$ factors in the expectation are erased. Then any corner-free subset of $S$ has size $o(|S|)$. Here a corner in $\mathbb{Z}_{N}^{2}$ is a set of the form $\{(x, y),(x+d, y),(x, y+d)\}$ for some $d \neq 0$. This claim follows from Theorem 3.1 by setting $Z=\mathbb{Z}_{N}, Z^{\prime}=\mathbb{Z}_{N}^{2}, \phi_{0}(d)=(0,0), \phi_{1}(d)=(d, 0)$, $\phi_{2}(d)=(0, d)$.

As in [Tao06a, Remark 2.19], we note that the hypothesis that $\left\{\phi_{i}(d)-\phi_{j}(d)\right.$ : $i, j \in J, d \in Z\}$ generate $Z^{\prime}$ can be dropped by foliating $Z^{\prime}$ into cosets. However, this results in a change to the linear forms hypothesis on $\nu$, namely, that it must be assumed on every coset.

We shall prove Theorem 3.1 by proving a somewhat more general removal-type result for arithmetic patterns. 
Theorem 3.3 (Relative arithmetic removal lemma). For every finite set $J$ and $\epsilon>0$, there exists $c>0$ so that the following holds. Let $Z, Z^{\prime},\left(\phi_{j}\right)_{j \in J}, \nu$ be the same as in Theorem 3.1. For any collection of functions $\left\{f_{j}: Z^{\prime} \rightarrow \mathbb{R}_{\geq 0}\right\}_{j \in J}$ satisfying $0 \leq f_{j}(x) \leq \nu(x)$ for all $x \in Z^{\prime}$ and $j \in J$, and such that

$$
\mathbb{E}\left[\prod_{j \in J} f_{j}\left(a+\phi_{j}(d)\right) \mid a \in Z^{\prime}, d \in Z\right] \leq c,
$$

one can find $A_{j} \subseteq Z^{\prime}$ for each $j \in J$ so that

$$
\prod_{j \in J} 1_{A_{j}}\left(a+\phi_{j}(d)\right)=0 \quad \text { for all } a \in Z^{\prime}, d \in Z
$$

and

$$
\mathbb{E}\left[f_{j}(x) 1_{Z^{\prime} \backslash A_{j}}(x) \mid x \in Z^{\prime}\right] \leq \epsilon \quad \text { for all } j \in J .
$$

Theorem 3.1 follows from Theorem 3.3 by setting $f_{j}=f$ for all $j \in J$ and $\epsilon<\delta /(r+1)$. Indeed, if the conclusion (8) fails, then Theorem 3.3 implies that there exists $A_{j} \subseteq Z^{\prime}$ for each $j \in J$ satisfying (10) and (11). The $A_{j}$ 's cannot have a common intersection, or else (10) fails for $d=0$. It follows that $\left\{Z^{\prime} \backslash A_{j}: j \in J\right\}$ covers $Z^{\prime}$, and hence (11) implies that $\mathbb{E}[f] \leq \sum_{j} \mathbb{E}\left[f_{j} 1_{Z^{\prime} \backslash A_{j}}\right] \leq(r+1) \epsilon<\delta$, which contradicts the hypothesis $\mathbb{E}[f] \geq \delta$.

Proof of Theorem 3.3. Let $V=\left(J,\left(V_{j}\right), r, H\right)$ be as in the statement of Theorem 3.1. Write $e_{j}:=J \backslash\{j\} \in H$. Define the weighted hypergraph $g$ on $V$ by setting

$$
g_{e_{j}}\left(x_{e_{j}}\right):=f_{j}\left(\psi_{j}\left(x_{e_{j}}\right)\right) \text { for all } j \in J
$$

where $\psi_{j}: V_{e_{j}} \rightarrow Z^{\prime}$ is defined by

$$
\psi_{j}\left(x_{e_{j}}\right)=\sum_{i \in e_{j}}\left(\phi_{i}\left(x_{i}\right)-\phi_{j}\left(x_{i}\right)\right)=a+\phi_{j}(d)
$$

where

$$
a=\sum_{i \in J} \phi_{i}\left(x_{i}\right) \quad \text { and } \quad d=-\sum_{i \in J} x_{i}
$$

Then, for all $x \in V$ and $a, d$ defined in (13), we have

$$
\prod_{j \in J} g_{e_{j}}\left(x_{e_{j}}\right)=\prod_{j \in J} f_{j}\left(a+\phi_{j}(d)\right)
$$

The homomorphism $x \mapsto(a, d): V \rightarrow Z^{\prime} \times Z$ given by (13) is surjective: the image contains $\left\{\left(\phi_{i}(d)-\phi_{j}(d), 0\right): i, j \in J, d \in Z\right\}$ and hence all of $Z^{\prime} \times\{0\}$. Moreover, the image also contains $\left\{\left(-\phi_{i}(d), d\right): i \in J, d \in Z\right\}$. Together, these sets generate 
all of $Z^{\prime} \times Z$. It follows that $(a, d)$ varies uniformly over $Z^{\prime} \times Z$ as $x$ varies uniformly over $V_{J}$, and so (14) implies that

$$
\mathbb{E}\left[\prod_{j \in J} g_{e_{j}}\left(x_{e_{j}}\right) \mid x \in V_{J}\right]=\mathbb{E}\left[\prod_{j \in J} f_{j}\left(a+\phi_{j}(d)\right) \mid a \in Z^{\prime}, d \in Z\right] \leq c .
$$

By the relative hypergraph removal lemma, for $c$ small enough (depending on $J$ and $\epsilon)$, we can find a subset $E_{j}^{\prime} \subset V_{e_{j}}$ for each $j \in J$ such that

$$
\prod_{j \in J} 1_{E_{j}^{\prime}}\left(x_{e_{j}}\right)=0 \quad \text { for all } x \in V_{J}
$$

and

$$
\mathbb{E}\left[g_{e_{j}}\left(x_{e_{j}}\right) 1_{V_{e_{j}} \backslash E_{j}^{\prime}}\left(x_{e_{j}}\right) \mid x_{e_{j}} \in V_{e_{j}}\right] \leq \epsilon /(r+1) \quad \text { for all } j \in J .
$$

For each $j \in J$, define $A_{j} \subseteq Z^{\prime}$ by

$$
A_{j}:=\left\{z^{\prime} \in Z^{\prime}:\left|\psi_{j}^{-1}\left(z^{\prime}\right) \cap E_{j}^{\prime}\right|>\frac{r}{r+1}\left|\psi_{j}^{-1}\left(z^{\prime}\right)\right|\right\} .
$$

In other words, $A_{j}$ contains $z^{\prime} \in Z^{\prime}$ if the hypergraph removal lemma removes less than a $1 /(r+1)$ fraction of the edges in $V_{e_{j}}$ representing $z^{\prime}$ via $\psi_{j}$.

For any $z^{\prime} \in Z^{\prime} \backslash A_{j}$, on the fiber $\psi^{-1}\left(z^{\prime}\right)$ the function $g_{e_{j}}$ takes the common value $f_{j}\left(z^{\prime}\right)$. Furthermore, by (16), on this fiber, the expectation of $1_{V_{e_{j}} \backslash E_{j}^{\prime}}$ is at least $1 /(r+1)$. Hence

$$
\mathbb{E}\left[f_{j}(x) 1_{Z^{\prime} \backslash A_{j}}(x) \mid x \in Z^{\prime}\right] \leq(r+1) \mathbb{E}\left[g_{e_{j}}\left(x_{e_{j}}\right) 1_{V_{e_{j}} \backslash E_{j}^{\prime}}\left(x_{e_{j}}\right) \mid x_{e_{j}} \in V_{e_{j}}\right] \leq \epsilon .
$$

This proves (11). To prove (10), suppose for some $a \in Z^{\prime}, d \in Z$ we have $a+\phi_{j}(d) \in$ $A_{j}$ for all $j \in J$. Let $V_{J}^{a, d} \subset V_{J}$ consist of all $x \in V_{J}$ satisfying (13). Then $\psi_{j}\left(x_{e_{j}}\right)=$ $a+\phi_{j}(d)$ for all $x \in V_{J}^{a, d}$ by (12), and in fact $\psi_{j}^{-1}\left(a+\phi_{j}(d)\right)$ is the projection of $V_{J}^{a, d}$ onto $V_{e_{j}}$. By (16), more than an $\frac{r}{r+1}$ fraction of this projection is in $E_{j}^{\prime}$. It follows by the pigeonhole principle (or a union bound on the complement) that there exists some $x \in V_{J}^{a, d}$ such that $x_{e_{j}} \in E_{j}^{\prime}$ for every $j \in J$. But this contradicts (15). Thus (10) holds.

\section{The Relative Hypergraph Removal Lemma}

Proof of Theorem 2.12. By Lemma 2.15, $\nu$ is upper o(1)-regular, so we can apply the weak sparse hypergraph regularity lemma (Theorem 2.16) to find functions $\tilde{g}_{e}$ : $V_{e} \rightarrow[0,1]$ for every $e \in H$ so that $(g, \tilde{g})$ is an $o(1)$-discrepancy pair. By the counting lemma (Theorem 2.17), we have

$$
\mathbb{E}\left[\prod_{e \in H} \tilde{g}_{e}\left(x_{e}\right) \mid x \in V_{J}\right]=\mathbb{E}\left[\prod_{e \in H} g_{e}\left(x_{e}\right) \mid x \in V_{J}\right]+o(1) \leq \delta+o(1) .
$$


The dense weighted hypergraph removal lemma (Theorem 2.11) tells us that for each $e \in H$ we can choose $E_{e}^{\prime} \subset V_{e}$ for which $V_{e} \backslash E_{e}^{\prime}$ has complexity $O_{\delta}(1)$ (i.e., at most some constant depending on $\delta$ ) and such that

$$
\prod_{e \in H} 1_{E_{e}^{\prime}}\left(x_{e}\right)=0 \quad \text { for all } x \in V_{J}
$$

and, as long as $\delta$ is small enough and $N$ is large enough, we have

$$
\mathbb{E}\left[\tilde{g}_{e}\left(x_{e}\right) 1_{V_{e} \backslash E_{e}^{\prime}}\left(x_{e}\right) \mid x_{e} \in V_{e}\right] \leq \epsilon / 2 \quad \text { for all } e \in H .
$$

As $V_{e} \backslash E_{e}^{\prime}$ has complexity $O_{\delta}(1)$, there is a partition of $V_{e} \backslash E_{e}^{\prime}$ into $O_{\delta}(1)$ hypergraphs $F_{e i}$ each of which is the set of $r$-cliques of some $(r-1)$-uniform hypergraph. We have

$$
\begin{aligned}
\left|\mathbb{E}\left[\left(\tilde{g}_{e}-g_{e}\right)\left(x_{e}\right) 1_{V_{e} \backslash E_{e}^{\prime}}\left(x_{e}\right) \mid x_{e} \in V_{e}\right]\right| & \leq \sum_{i}\left|\mathbb{E}\left[\left(\tilde{g}_{e}-g_{e}\right)\left(x_{e}\right) 1_{F_{e i}}\left(x_{e}\right) \mid x_{e} \in V_{e}\right]\right| \\
& \leq \sum_{i} o(1)=O_{\delta}(1) o(1) \leq \epsilon / 2 \text { for all } e \in H .
\end{aligned}
$$

We used that $\left(g_{e}, \tilde{g}_{e}\right)$ is an $o(1)$-discrepancy pair on each of the terms of the sum, and the final inequality is true as long as $N$ is large enough. Combining (17) and (18) we obtain

$$
\mathbb{E}\left[g_{e}\left(x_{e}\right) 1_{V_{e} \backslash E_{e}^{\prime}}(x) \mid x_{e} \in V_{e}\right] \leq \epsilon \quad \text { for all } e \in H .
$$

This proves the claim.

\section{The Weak Regularity Lemma}

Let $X$ be a finite set and $g: X \rightarrow \mathbb{R}_{\geq 0}$. Let $\mathcal{F}$ be a family of subsets of $X$ which is closed under intersection, $X \in \mathcal{F}$, all subsets of $X$ of size one are in $\mathcal{F}$, and such that, for every $S \in \mathcal{F}$, there is a partition of $X$ which contains $S$ and consists of members of $\mathcal{F}$. For $t \geq 2$, the family $\mathcal{F}$ is $t$-splittable if for every $S \in \mathcal{F}$ there is a partition $P$ of $X$ into members of $\mathcal{F}$ such that $S \in P$ and $|P| \leq t$. The complexity $p=p(f)$ of a function $f: X \rightarrow \mathbb{R}_{\geq 0}$ is the minimum $p$ for which there is a partition $X=S_{1} \cup \cdots \cup S_{p}$ into $p$ subsets each in $\mathcal{F}$ such that $f$ is constant on each $S_{i}$. We call $(g, \tilde{g})$ an $\epsilon$-discrepancy pair if for all $A \in \mathcal{F}$,

$$
\left|\mathbb{E}\left[(g-\tilde{g}) 1_{A}\right]\right| \leq \epsilon
$$

All expectations are taken with the uniform measure on $X$. For $P$ a partition of $X$, let $g_{P}$ be the function on $X$ given by $g_{P}(x)=\frac{\mathbb{E}\left[g 1_{A}\right]}{\mathbb{E}\left[1_{A}\right]}$ when $x \in A \in P$. That is, $g_{P}(x)$ is the conditional expectation of $g(x)$ given the partition $P$ and is constant on any part $A$ of the partition. 
The function $g$ we call upper $\eta$-regular if for every $A \in \mathcal{F}$, we have

$$
\mathbb{E}\left[g 1_{A}\right] \leq \mathbb{E}\left[1_{A}\right]+\eta .
$$

If $g$ is upper $\eta$-regular, $A, B \in \mathcal{F}$, and $\mathcal{F}$ is $t$-splittable, then

$$
\mathbb{E}\left[g 1_{B \backslash A}\right] \leq \mathbb{E}\left[1_{B \backslash A}\right]+(t-1) \eta .
$$

Indeed, in this case $B \backslash A$ can be partitioned into $t-1$ sets in $\mathcal{F}$ (we first split with respect to $A$ and then consider the intersections of the parts of the partition with $B)$. Applying the upper $\eta$-regularity condition to each of these sets and summing up the inequalities, we arrive at (19).

Following Scott [Sco11], let $\phi: \mathbb{R}_{\geq 0} \rightarrow \mathbb{R}_{\geq 0}$ be the convex function given by

$$
\phi(u)= \begin{cases}u^{2} & \text { if } u \leq 2, \\ 4 u-4 & \text { otherwise. }\end{cases}
$$

For a partition $P$ of $X$, let $\phi(P)=\mathbb{E}\left[\phi\left(g_{P}\right)\right]$, which is the mean $\phi$-density of $g$ with respect to the partition $P$. As $\phi$ takes only nonnegative values and $\phi(u) \leq 4 u$, we have

$$
0 \leq \phi(P) \leq 4 \mathbb{E}\left[g_{P}\right]=4 \mathbb{E}[g] .
$$

Also, by the convexity of $\phi$, it follows that if $P^{\prime}$ is a refinement of $P$, then $\phi\left(P^{\prime}\right) \geq$ $\phi(P)$.

Lemma 5.1. Let $X$ and $\mathcal{F}$ as above be such that $\mathcal{F}$ is $t$-splittable. Let $0<\epsilon, \eta<1$ and $T=t^{20 / \epsilon^{2}}$. For any $g: X \rightarrow \mathbb{R}_{\geq 0}$ which is upper $\eta$-regular with $\eta \leq \frac{\epsilon}{8 t T}$, there is $\tilde{g}: X \rightarrow[0,1]$ with complexity at most $T$ such that $(g, \tilde{g})$ is an $\epsilon$-discrepancy pair.

Proof. Let $\alpha=\frac{\epsilon^{2}}{4}$. We first find a partition $P$ of $X$ into members of $\mathcal{F}$ with $|P| \leq$ $t^{5 / \alpha}=T$ such that for any refinement $P^{\prime}$ of $P$ into members of $\mathcal{F}$ with $\left|P^{\prime}\right| \leq t|P|$, we have $\phi\left(P^{\prime}\right)-\phi(P)<\alpha$. In order to construct $P$, we first recursively construct a sequence $P_{0}, P_{1}, \ldots$ of finer partitions of $X$ into members of $\mathcal{F}$ so that $\left|P_{j}\right| \leq t^{j}$ and $\phi\left(P_{j}\right) \geq j \alpha$. We begin by considering the trivial partition $P_{0}=\{X\}$, which satisfies $\phi\left(P_{0}\right) \geq 0$. At the beginning of step $j+1$, we have a partition $P_{j}$ of $X$ into members of $\mathcal{F}$ with $\left|P_{j}\right| \leq t^{j}$ and $\phi\left(P_{j}\right) \geq j \alpha$. If there exists a refinement $P_{j+1}$ of $X$ into members of $\mathcal{F}$ with $\left|P_{j+1}\right| \leq t\left|P_{j}\right|$ and $\phi\left(P_{j+1}\right) \geq \phi\left(P_{j}\right)+\alpha$, then we continue to step $j+2$. Otherwise, we may pick $P=P_{j}$ to be the desired partition. Note that this process must stop after at most $5 / \alpha$ steps since $5>4(1+\eta) \geq 4 \mathbb{E}[g] \geq \phi\left(P_{j}\right) \geq j \alpha$, where the second inequality follows from $g$ being upper $\eta$-regular. We therefore arrive at the desired partition $P$.

Let $P$ be $X=S_{1} \cup \cdots \cup S_{p}$. Let $\tilde{g}: X \rightarrow[0,1]$, where $\tilde{g}=g_{P} \wedge 1$ is the minimum of $g_{P}$ and the constant function 1 . We will show that $\left(g_{P}, \tilde{g}\right)$ is an $\frac{\epsilon}{4}$-discrepancy pair and $\left(g_{P}, g\right)$ is a $\frac{3 \epsilon}{4}$-discrepancy pair, which implies by the triangle inequality that $(g, \tilde{g})$ is an $\epsilon$-discrepancy pair. As $\tilde{g}$ has complexity at most $|P| \leq T$, this will complete the proof. 
We first show $\left(g_{P}, \tilde{g}\right)$ is an $\frac{\epsilon}{4}$-discrepancy pair. Note that $g_{P}-\tilde{g}$ is nonnegative and constant on each part of $P$. If $S_{i} \in P$ and $g_{P}-\tilde{g}>0$ on $S_{i}$, then also $g_{P}>1$ and $\tilde{g}=1$ on $S_{i}$. As $g$ is upper $\eta$-regular, we have $\mathbb{E}\left[g 1_{S_{i}}\right] \leq \mathbb{E}\left[1_{S_{i}}\right]+\eta$ and hence $\mathbb{E}\left[(g-\tilde{g}) 1_{S_{i}}\right] \leq \eta$. Therefore, by summing over all parts in the partition $P$, we see that if $A \in \mathcal{F}$,

$$
0 \leq \mathbb{E}\left[\left(g_{P}-\tilde{g}\right) 1_{A}\right] \leq \mathbb{E}\left[\left(g_{P}-\tilde{g}\right)\right] \leq \eta|P| \leq \eta T \leq \frac{\epsilon}{4},
$$

and $\left(g_{P}, \tilde{g}\right)$ is an $\frac{\epsilon}{4}$-discrepancy pair.

We next show that $\left(g_{P}, g\right)$ is a $\frac{3 \epsilon}{4}$-discrepancy pair, which completes the proof. Suppose for contradiction that there is $A \in \mathcal{F}$ such that

$$
\left|\mathbb{E}\left[\left(g_{P}-g\right) 1_{A}\right]\right|>\frac{3 \epsilon}{4} .
$$

Let $B$ be the union of all $S_{i} \cap A$, where $S_{i} \in P$, for which both $\mathbb{E}\left[1_{S_{i} \cap A}\right] \geq t \eta$ and $\mathbb{E}\left[1_{S_{i} \backslash A}\right] \geq t \eta$.

We claim that for each $S_{i} \in P$, we have

$$
\left|\mathbb{E}\left[\left(g_{P}-g\right)\left(1_{A \cap S_{i}}-1_{B \cap S_{i}}\right)\right]\right| \leq 2 t \eta .
$$

Indeed, if $B \cap S_{i}=A \cap S_{i}$, then the left hand side of (20) is 0. Otherwise, $\mathbb{E}\left[1_{A \cap S_{i}}\right] \leq t \eta$ or $\mathbb{E}\left[1_{S_{i} \backslash A}\right] \leq t \eta$. In the first case, when $\mathbb{E}\left[1_{A \cap S_{i}}\right] \leq t \eta$, we have $1_{B \cap S_{i}}$ is identically 0 , as well as

$$
\mathbb{E}\left[g 1_{A \cap S_{i}}\right] \leq \mathbb{E}\left[1_{A \cap S_{i}}\right]+\eta \leq(t+1) \eta
$$

and

$$
\mathbb{E}\left[g_{P} 1_{A \cap S_{i}}\right]=\frac{\mathbb{E}\left[g 1_{S_{i}}\right]}{\mathbb{E}\left[1_{S_{i}}\right]} \mathbb{E}\left[1_{A \cap S_{i}}\right] \leq \frac{\left(\mathbb{E}\left[1_{S_{i}}\right]+\eta\right)}{\mathbb{E}\left[1_{S_{i}}\right]} \mathbb{E}\left[1_{A \cap S_{i}}\right] \leq \mathbb{E}\left[1_{A \cap S_{i}}\right]+\eta \leq(t+1) \eta,
$$

from which (20) follows. In the second case, when $\mathbb{E}\left[1_{S_{i} \backslash A}\right] \leq t \eta$, we again have $1_{B \cap S_{i}}$ is identically 0 , so that

$$
\begin{aligned}
\mathbb{E}\left[\left(g-g_{P}\right)\left(1_{A \cap S_{i}}-1_{B \cap S_{i}}\right)\right] & =\mathbb{E}\left[\left(g-g_{P}\right) 1_{A \cap S_{i}}\right]=\mathbb{E}\left[\left(g-g_{P}\right)\left(1_{S_{i}}-1_{S_{i} \backslash A}\right)\right] \\
& =\mathbb{E}\left[\left(g-g_{P}\right) 1_{S_{i}}\right]-\mathbb{E}\left[\left(g-g_{P}\right) 1_{S_{i} \backslash A}\right]=-\mathbb{E}\left[\left(g-g_{P}\right) 1_{S_{i} \backslash A}\right],
\end{aligned}
$$

and similar to the first case, using (19) to estimate $\mathbb{E}\left[g 1_{S_{i} \backslash A}\right]$ and $\mathbb{E}\left[g_{P} 1_{S_{i} \backslash A}\right]$, we get (20).

Notice that

$$
\left|\mathbb{E}\left[\left(g_{P}-g\right) 1_{A}\right]-\mathbb{E}\left[\left(g_{P}-g\right) 1_{B}\right]\right|=\left|\mathbb{E}\left[\left(g_{P}-g\right)\left(1_{A}-1_{B}\right)\right]\right| \leq|P| 2 t \eta \leq \frac{\epsilon}{4},
$$

where the first inequality follows by using (20) for each part $S_{i}$ and the triangle inequality. Hence,

$$
\left|\mathbb{E}\left[\left(g_{P}-g\right) 1_{B}\right]\right| \geq\left|\mathbb{E}\left[\left(g_{P}-g\right) 1_{A}\right]\right|-\left|\mathbb{E}\left[\left(g_{P}-g\right) 1_{A}\right]-\mathbb{E}\left[\left(g_{P}-g\right) 1_{B}\right]\right|>\frac{3 \epsilon}{4}-\frac{\epsilon}{4}=\frac{\epsilon}{2} .
$$


Let $\hat{P}$ be the refinement of $P$ where $S_{i}$ is also in $\hat{P}$ if $B \cap S_{i}=\emptyset$ and otherwise $S_{i} \cap B$ and $S_{i} \backslash B$ are parts of $\hat{P}$, and let $P^{\prime}$ be a refinement of $\hat{P}$ into at most $t|P|$ members of $\mathcal{F}$. The refinement $P^{\prime}$ exists as $\mathcal{F}$ is $t$-splittable and is closed under intersections, $P$ consists of members of $\mathcal{F}, A \in \mathcal{F}$, and $S_{i} \cap B=S_{i} \cap A \in \mathcal{F}$ if $S_{i} \cap B \in \hat{P}$. As $P^{\prime}$ is a refinement of $\hat{P}$ which is a refinement of $P$, we have $\phi\left(P^{\prime}\right) \geq \phi(\hat{P}) \geq \phi(P)$. Let $R \in\left\{S_{i}, S_{i} \cap B, S_{i} \backslash B\right\}$, where $S_{i}$ is a part of $P$ that is refined into two parts in $\hat{P}$, so that $\mathbb{E}\left[1_{R}\right] \geq t \eta$. Letting $u=\frac{\mathbb{E}\left[g 1_{R}\right]}{\mathbb{E}\left[1_{R}\right]}$, we see, since $g$ is upper $\eta$-regular and using (19), that $u \leq 1+t \eta(t \eta)^{-1}=2$ and hence $\phi(u)=u^{2}$. It follows, by considering the functions pointwise, that $\phi\left(g_{\hat{P}}\right)-\phi\left(g_{P}\right)=g_{\hat{P}}^{2}-g_{P}^{2}$. Hence,

$$
\begin{aligned}
\phi\left(P^{\prime}\right)-\phi(P) & \geq \phi(\hat{P})-\phi(P)=\mathbb{E}\left[g_{\hat{P}}^{2}\right]-\mathbb{E}\left[g_{P}^{2}\right]=\mathbb{E}\left[g_{\hat{P}}^{2}-g_{P}^{2}\right]=\mathbb{E}\left[\left(g_{\hat{P}}-g_{P}\right)^{2}\right] \\
& \geq \mathbb{E}\left[\left(g_{\hat{P}}-g_{P}\right) 1_{B}\right]^{2}=\mathbb{E}\left[\left(g-g_{P}\right) 1_{B}\right]^{2}>\frac{\epsilon^{2}}{4}=\alpha .
\end{aligned}
$$

The third equality above is the Pythagorean identity, which uses that $\hat{P}$ is a refinement of $P$, and the second inequality is an application of the Cauchy-Schwarz inequality. However, since $P^{\prime}$ is a refinement of $P$ consisting of members of $\mathcal{F}$ with $\left|P^{\prime}\right| \leq t|P|$, this contradicts $\phi\left(P^{\prime}\right)-\phi(P)<\alpha$ from the definition of $P$ and completes the proof.

To establish the weak hypergraph regularity lemma, Theorem 2.16, we use Lemma 5.1 with $X=V_{1} \times \cdots \times V_{r}$ and $\mathcal{F}$ being the family of subsets of $X$ which form the $r$-cliques of some $r$-partite $(r-1)$-uniform hypergraph with parts $V_{1}, \ldots, V_{r}$. Noting that $\mathcal{F}$ is $2^{r}$-splittable in this case, we obtain Theorem 2.16.

\section{The Counting Lemma}

The three main ingredients in our proof of the counting lemma (Theorem 2.17) are as follows.

(1) A standard telescoping argument [BCLSV08] in the dense case, i.e., when $\nu=1$.

(2) Repeated applications of the Cauchy-Schwarz inequality. This is a standard technique in this area, e.g., [Gow01, Gow07, GT08, Tao06a].

(3) Densification. This is the main new ingredient in our proof. At each step, we reduce the problem of counting $H$ in a particular weighted hypergraph to that of counting $H$ in a modified weighted hypergraph. For an edge $e \in H$, we replace the triple $\left(\nu_{e}, g_{e}, \tilde{g}_{e}\right)$ by a new triple $\left(1, g_{e}^{\prime}, \tilde{g}_{e}^{\prime}\right)$ with $0 \leq g_{e}^{\prime}, \tilde{g}_{e}^{\prime} \leq 1$ and such that $\left(g_{e}^{\prime}, \tilde{g}_{e}^{\prime}\right)$ is an $\epsilon^{\prime}$-discrepancy pair for some $\epsilon^{\prime}=o_{\epsilon \rightarrow 0}(1)$. By repeatedly applying this reduction to all $e \in H$ (we use induction), we reduce the counting lemma to the dense case.

We developed the densification technique in our earlier paper [CFZ14], where we proved a sparse counting lemma in graphs. We have significantly simplified a number of technical steps from [CFZ14] in order to extend the densification technique to hypergraphs here. 
6.1 Telescoping argument. The following argument allows us to prove the counting lemma in the dense case, i.e., when $0 \leq g \leq 1$.

LEMma 6.1 (Telescoping discrepancy argument for dense hypergraphs). Theorem 2.17 holds if we assume that there is some $e_{1} \in H$ so that $\nu_{e}=1$ for all $e \in H \backslash\left\{e_{1}\right\}$. In fact, in this case,

$$
\left|\mathbb{E}\left[\prod_{e \in H} g_{e}\left(x_{e}\right) \mid x \in V_{J}\right]-\mathbb{E}\left[\prod_{e \in H} \tilde{g}_{e}\left(x_{e}\right) \mid x \in V_{J}\right]\right| \leq|H| \epsilon .
$$

Lemma 6.1 uses only the assumption that $\left(g_{e}, \tilde{g}_{e}\right)$ is an $\epsilon$-discrepancy pair for every $e \in H$ and nothing about the linear forms condition on $\nu$. Recall that for each fixed $e \in H$, the condition that $\left(g_{e}, \tilde{g}_{e}\right)$ is an $\epsilon$-discrepancy pair means that for all subsets $B_{f} \subseteq V_{f}, f \in \partial e$, we have

$$
\left|\mathbb{E}\left[\left(g_{e}\left(x_{e}\right)-\tilde{g}_{e}\left(x_{e}\right)\right) \prod_{f \in \partial e} 1_{B_{f}}\left(x_{f}\right) \mid x_{e} \in V_{e}\right]\right| \leq \epsilon .
$$

This is equivalent to the condition that for all functions $u_{f}: V_{f} \rightarrow[0,1], f \in \partial e$, we have

$$
\left|\mathbb{E}\left[\left(g_{e}\left(x_{e}\right)-\tilde{g}_{e}\left(x_{e}\right)\right) \prod_{f \in \partial e} u_{f}\left(x_{f}\right) \mid x_{e} \in V_{e}\right]\right| \leq \epsilon .
$$

Indeed, the expectation is linear in each $u_{f}$ and hence the extrema occur when the $u_{f}$ 's are $\{0,1\}$-valued, thereby reducing to $(22)$.

Proof. Let $h=|H|$ and order the edges of $H \backslash\left\{e_{1}\right\}$ arbitrarily as $e_{2}, \ldots, e_{h}$. We can write the left-hand side of (21), without the absolute values, as a telescoping sum

$$
\sum_{t=1}^{h} \mathbb{E}\left[\left(\prod_{s=1}^{t-1} \tilde{g}_{e_{s}}\left(x_{e_{s}}\right)\right)\left(g_{e_{t}}\left(x_{e_{t}}\right)-\tilde{g}_{e_{t}}\left(x_{e_{t}}\right)\right)\left(\prod_{s=t+1}^{h} g_{e_{s}}\left(x_{e_{s}}\right)\right) \mid x \in V_{J}\right] .
$$

For the $t$-th term in the sum, when we fix the value of $x_{J \backslash e_{t}} \in V_{J \backslash e_{t}}$, the expectation has the form

$$
\mathbb{E}\left[\left(g_{e_{t}}\left(x_{e_{t}}\right)-\tilde{g}_{e_{t}}\left(x_{e_{t}}\right)\right) \prod_{f \in \partial e_{t}} u_{f}\left(x_{f}\right) \mid x_{e_{t}} \in V_{e_{t}}\right]
$$

for some functions $u_{f}: V_{f} \rightarrow[0,1]$ (here we used the key fact that $g_{e_{s}} \leq 1$ for all $s>1$ and $\tilde{g}_{e_{s}} \leq 1$ for all $\left.s\right)$. Since $\left(g_{e_{t}}, \tilde{g}_{e_{t}}\right)$ is an $\epsilon$-discrepancy pair, (23) implies that $(25)$ is bounded in absolute value by $\epsilon$. The same bound holds after we vary $x_{J \backslash e_{t}} \in V_{J \backslash e_{t}}$. So every term in (24) is bounded by $\epsilon$ in absolute value, and hence (24) is at most $h \epsilon$ in absolute value. 
6.2 Strong linear forms. The main result of this subsection tells us that $\nu$ can be replaced by the constant function 1 in counting expressions. Though somewhat technical in detail, the main idea of the proof is quite simple and may be summarized as follows: we use the Cauchy-Schwarz inequality to double each vertex $j$ of a certain edge in turn, at each step majorizing those edges which do not contain $j$. This method is quite standard in the field. In the work of Green and Tao, it is used to prove generalized von Neumann theorems [GT08, Prop. 5.3], [Tao06a, Thm. 3.8], although the statement of our lemma is perhaps more similar to the uniform distribution property [GT08, Prop. 6.2], [Tao06a, Prop. 5.1].

We begin by using a similar method to prove a somewhat easier result. It shows that if $\nu$ satisfies the $H$-linear forms condition then $(\nu, 1)$ is an $o(1)$-discrepancy pair, which implies Lemma 2.15.

LEmma 6.2. Let $e$ be a finite set, $V_{j}$ a finite set for each $j \in e$, and $V_{e}=\prod_{j \in e} V_{j}$. Then, for any function $\nu: V_{e} \rightarrow \mathbb{R}$ and any collection of $B_{f} \subseteq V_{f}$ for $f \in \partial e$,

$\left|\mathbb{E}\left[\left(\nu_{e}\left(x_{e}\right)-1\right) \prod_{f \in \partial e} 1_{B_{f}}\left(x_{f}\right) \mid x_{e} \in V_{e}\right]\right| \leq \mathbb{E}\left[\prod_{\omega \in\{0,1\} e}\left(\nu_{e}\left(x_{e}^{(\omega)}\right)-1\right) \mid x_{e}^{(0)}, x_{e}^{(1)} \in V_{e}\right]^{1 / 2^{|e|}}$.

Lemma 6.2 follows from a direct application of the Gowers-CauchySchwarz [Gow01] inequality for hypergraphs (see [CFZ]). We include the proof here for completeness.

Proof. For $\emptyset \subseteq d \subseteq e$, let

$$
X_{d}:=\prod_{\omega \in\{0,1\}^{d}}\left(v_{e}\left(x_{e \backslash d}, x_{d}^{(\omega)}\right)-1\right), \quad Y_{d}:=\prod_{\substack{f \in \partial e \\ f \supseteq d}} \prod_{\omega \in\{0,1\}^{d}} 1_{B_{f}}\left(x_{f \backslash d}, x_{d}^{(\omega)}\right),
$$

and

$$
Q_{d}:=\mathbb{E}\left[X_{d} Y_{d} \mid x_{e \backslash d} \in V_{e \backslash d}, x_{d}^{(0)}, x_{d}^{(1)} \in V_{d}\right] .
$$

Then (26) can be written as $\left|Q_{\emptyset}\right| \leq Q_{e}^{1 / 2^{|e|}}$. By induction, it suffices to show that $Q_{d}^{2} \leq Q_{d \cup\{j\}}$ whenever $j \in e \backslash d$. Let $Y_{d}=Y_{d}^{\ni j} Y_{d}^{\not j}$ where $Y_{d}^{\ni j}$ consists of all the factors in $Y_{d}$ that contain $x_{j}$ in the argument, and $Y_{d}^{\not j}$ consists of all other factors. By the Cauchy-Schwarz inequality, we have

$$
Q_{d}^{2}=\mathbb{E}\left[\mathbb{E}\left[X_{d} Y_{d}^{\ni j} \mid x_{j} \in V_{j}\right] Y_{d}^{\not \ni j}\right]^{2} \leq \mathbb{E}\left[\mathbb{E}\left[X_{d} Y_{d}^{\ni j} \mid x_{j} \in V_{j}\right]^{2}\right] \mathbb{E}\left[\left(Y_{d}^{\not \supset j}\right)^{2}\right] \leq Q_{d \cup\{j\}},
$$

since $Q_{d \cup\{j\}}=\mathbb{E}\left[\mathbb{E}\left[X_{d} Y_{d}^{\ni j} \mid x_{j} \in V_{j}\right]^{2}\right]$ and $0 \leq Y_{d}^{\nexists j} \leq 1$, where the outer expectations are taken over all free variables. This shows that $Q_{d}^{2} \leq Q_{d \cup\{j\}}$. Hence, $\left|Q_{\emptyset}\right| \leq Q_{e^{1 / 2^{|e|}}}$, as desired.

The next lemma is very similar, except that now we need to invoke the linear forms condition. 
LEMMA 6.3 (Strong linear forms). Let $V=\left(J,\left(V_{j}\right)_{j \in J}, r, H\right)$ be a hypergraph system and let $\nu$ be a weighted hypergraph on $V$ satisfying the linear forms condition. Let $e_{1} \in H$. For each $\iota \in\{0,1\}$ and $e \in H \backslash\left\{e_{1}\right\}$, let $g_{e}^{(\iota)}: V_{e} \rightarrow \mathbb{R}_{\geq 0}$ be a function so that either $g_{e}^{(\iota)} \leq 1$ or $g_{e}^{(\iota)} \leq \nu_{e}$ holds. Then

$\mathbb{E}\left[\left(\nu_{e_{1}}\left(x_{e_{1}}\right)-1\right) \prod_{\iota \in\{0,1\}}\left(\prod_{e \in H \backslash\left\{e_{1}\right\}} g_{e}^{(\iota)}\left(x_{e}^{(\iota)}\right)\right) \mid x_{J}^{(0)}, x_{J}^{(1)} \in V_{J} ; x_{e_{1}}^{(0)}=x_{e_{1}}^{(1)}=x_{e_{1}}\right]=o(1)$.

In (27), the notation $x_{e_{1}}^{(0)}=x_{e_{1}}^{(1)}=x_{e_{1}}$ means that $x_{j}^{(0)}, x_{j}^{(1)}, x_{j}$ are taken to be the same for all $j \in e_{1}$. Recall that we write $o(1)$ for a quantity that tends to zero as $N \rightarrow \infty$.

Proof. For each $\iota \in\{0,1\}$ and $e \in H \backslash\left\{e_{1}\right\}$, let $\bar{g}_{e}^{(\iota)}$ be either 1 or $\nu_{e}$ so that $g_{e}^{(\iota)} \leq \bar{g}_{e}^{(\iota)}$ holds. For $\emptyset \subseteq d \subseteq e_{1}$, define

$$
\begin{aligned}
X_{d} & :=\prod_{\omega \in\{0,1\}^{d}}\left(\nu_{e_{1}}\left(x_{e_{1} \backslash d}, x_{d}^{(\omega)}\right)-1\right), \\
Y_{d} & :=\prod_{\iota \in\{0,1\}} \prod_{e \in H \backslash\left\{e_{1}\right\}} \prod_{\omega \in\{0,1\}^{e \cap d}}\left\{\begin{array}{ll}
g_{e}^{(\iota)}\left(x_{e \backslash e_{1}}^{(\iota)}, x_{d}^{(\omega)}, x_{e \cap e_{1} \backslash d}\right) & \text { if } e \supseteq d \\
\bar{g}_{e}^{(\iota)}\left(x_{e \backslash e_{1}}^{(\iota)}, x_{e \cap d}^{(\omega)}, x_{e \cap e_{1} \backslash d}\right) & \text { if } e \nsupseteq d
\end{array}\right\},
\end{aligned}
$$

and

$$
Q_{d}:=\mathbb{E}\left[X_{d} Y_{d} \mid x_{\left(J \backslash e_{1}\right) \cup d}^{(0)}, x_{\left(J \backslash e_{1}\right) \cup d}^{(1)} \in V_{\left(J \backslash e_{1}\right) \cup d}, x_{e_{1} \backslash d} \in V_{e_{1} \backslash d}\right] .
$$

We observe that $Q_{\emptyset}$ is equal to the left-hand side of (27) and

$$
\begin{aligned}
Q_{e_{1}}=\mathbb{E} & {\left[\prod_{\omega \in\{0,1\}^{e_{1}}}\left(\nu_{e_{1}}\left(x_{e_{1}}^{(\omega)}\right)-1\right) \prod_{\iota \in\{0,1\}} \prod_{e \in H \backslash\left\{e_{1}\right\}} \prod_{\omega \in\{0,1\}\}_{e n e_{1}}} \bar{g}_{e}^{(\iota)}\left(x_{e \backslash e_{1}}^{(\iota)}, x_{e \cap e_{1}}^{(\omega)}\right)\right.} \\
& \left.\mid x_{J}^{(0)}, x_{J}^{(1)} \in V_{J}\right]=o(1)
\end{aligned}
$$

by the linear forms condition (4). Indeed, after we expand $\prod_{\omega \in\{0,1\}^{e_{1}}}\left(\nu_{e_{1}}\left(x_{e_{1}}^{(\omega)}\right)-1\right)$, every term in $Q_{e_{1}}$ has the form of (4) (since $\bar{g}_{e}^{(\iota)}$ is 1 or $\nu_{e}$ ). Thus $Q_{e_{1}}$ is the sum of $2^{\left|e_{1}\right|}$ terms, each of which is $\pm(1+o(1))$ by the linear forms condition, and they cancel accordingly to $o(1)$.

We claim that if $j \in e_{1} \backslash d$ then

$$
\left|Q_{d}\right| \leq(1+o(1)) Q_{d \cup\{j\}}^{1 / 2},
$$

4 This is where the weak 2-blow-up of $H$ arises, since the estimate $Q_{e_{1}}=o(1)$ only relies upon knowing that $\nu$ has roughly the expected density for certain subgraphs of the weak 2-blow-up. 
from which it would follow by induction that

$$
\mid \text { LHS of }(27)|=| Q_{\emptyset} \mid \leq(1+o(1)) Q_{e_{1}}^{1 / 2^{r}}=o(1) .
$$

Now we prove (28). Let $Y_{d}=Y_{d}^{\ni j} Y_{d}^{\ngtr j}$ where $Y_{d}^{\ni j}$ consists of all the factors in $Y_{d}$ that contain $x_{j}$ in the argument, and $Y_{d}^{\ngtr j}$ consists of all other factors. Using the CauchySchwarz inequality and $Y_{d}^{\not j} \leq \bar{Y}_{d}^{\not j j}$, where $\bar{Y}_{d}^{\not j}$ denotes the expression obtained from $Y_{d}^{\not j}$ by replacing all $g$ 's by $\bar{g}$ 's, one has

$$
\begin{aligned}
Q_{d}^{2} & =\mathbb{E}\left[\mathbb{E}\left[X_{d} Y_{d}^{\ni j} \mid x_{j} \in V_{j}\right] Y_{d}^{\not j}\right]^{2} \leq \mathbb{E}\left[\mathbb{E}\left[X_{d} Y_{d}^{\ni j} \mid x_{j} \in V_{j}\right]^{2} Y_{d}^{\not j j}\right] \mathbb{E}\left[Y_{d}^{\not j}\right] \\
& \leq \mathbb{E}\left[\mathbb{E}\left[X_{d} Y_{d}^{\ni j} \mid x_{j} \in V_{j}\right]^{2} \bar{Y}_{d}^{\not j}\right] \mathbb{E}\left[\bar{Y}_{d}^{\ngtr j}\right]=Q_{d \cup\{j\}} \mathbb{E}\left[\bar{Y}_{d}^{\ngtr j}\right]
\end{aligned}
$$

where the outer expectations are taken over all free variables. The second factor in (29) is $1+o(1)$ by the linear forms condition (4) as $\bar{Y}_{d}^{\ngtr j}$ consists only of $\nu$ factors. This proves (28).

6.3 Counting lemma proof. As already mentioned, the main idea of the following proof is a process called densification, where we reduce the problem of counting $H$ in a sparse hypergraph to that of counting $H$ in a dense hypergraph by replacing sparse edges with dense edges one at a time. Several steps are needed to densify a given edge $e_{1}$. The first step is to double all vertices outside of $e_{1}$ and to majorize $g_{e_{1}}$ by $\nu_{e_{1}}$. We then use the strong linear forms condition to remove the edge corresponding to $e_{1}$ entirely. This leaves us with the seemingly harder problem of counting the graph $H^{\prime}$ consisting of two copies of $H \backslash\left\{e_{1}\right\}$ joined along the vertices of $e_{1}$. However, an inductive hypothesis tells us that we can count copies of $H \backslash\left\{e_{1}\right\}$. The core of the proof is in showing that this allows us to replace one of the copies of $H \backslash\left\{e_{1}\right\}$ in $H^{\prime}$ by a dense edge, thus reducing our problem to that of counting $H$ with one edge replaced by a dense edge.

Proof of Theorem 2.17. We use induction on $\left|\left\{e \in H: \nu_{e} \neq 1\right\}\right|$. When $\left|\left\{e \in H: \nu_{e} \neq 1\right\}\right|=0$ or 1 , the result follows from Lemma 6.1. Now take $e_{1} \in H$ so that $\nu_{e_{1}} \neq 1$.

We assume that $|J|$ is a fixed constant. We write $o(1)$ for a quantity that tends to zero as $N \rightarrow \infty$ and $o_{\epsilon \rightarrow 0}(1)$ for a quantity that tends to zero as $N \rightarrow \infty$ and $\epsilon \rightarrow 0$. We need to show that the following quantity is $o_{\epsilon \rightarrow 0}(1)$ :

$$
\begin{aligned}
\mathbb{E}\left[\prod_{e \in H} g_{e}\left(x_{e}\right) \mid x \in V_{J}\right]-\mathbb{E}\left[\prod_{e \in H} \tilde{g}_{e}\left(x_{e}\right) \mid x \in V_{J}\right] \\
=\mathbb{E}\left[g_{e_{1}}\left(x_{e_{1}}\right)\left(\prod_{e \in H \backslash\left\{e_{1}\right\}} g_{e}\left(x_{e}\right)-\prod_{e \in H \backslash\left\{e_{1}\right\}} \tilde{g}_{e}\left(x_{e}\right)\right) \mid x \in V_{J}\right] \\
+\mathbb{E}\left[\left(g_{e_{1}}\left(x_{e_{1}}\right)-\tilde{g}_{e_{1}}\left(x_{e_{1}}\right)\right)\left(\prod_{e \in H \backslash\left\{e_{1}\right\}} \tilde{g}_{e}\left(x_{e}\right)\right) \mid x \in V_{J}\right] .
\end{aligned}
$$


The second term on the right-hand side of (30) is at most $\epsilon$ in absolute value since $\left(g_{e_{1}}, \tilde{g}_{e_{1}}\right)$ is an $\epsilon$-discrepancy pair and $\tilde{g} \leq 1$ (e.g., see proof of Lemma 6.1). It remains to show that the first term on the right-hand side of $(30)$ is $o_{\epsilon \rightarrow 0}(1)$.

Define functions $\nu_{e_{1}}^{\prime}, g_{e_{1}}^{\prime}, \tilde{g}_{e_{1}}^{\prime}: V_{e_{1}} \rightarrow \mathbb{R}_{\geq 0}$ by

$$
\begin{aligned}
& \nu_{e_{1}}^{\prime}\left(x_{e_{1}}\right):=\mathbb{E}\left[\prod_{e \in H \backslash\left\{e_{1}\right\}} \nu_{e}\left(x_{e}\right) \mid x_{J \backslash e_{1}} \in V_{J \backslash e_{1}}\right], \\
& g_{e_{1}}^{\prime}\left(x_{e_{1}}\right):=\mathbb{E}\left[\prod_{e \in H \backslash\left\{e_{1}\right\}} g_{e}\left(x_{e}\right) \mid x_{J \backslash e_{1}} \in V_{J \backslash e_{1}}\right], \\
& \tilde{g}_{e_{1}}^{\prime}\left(x_{e_{1}}\right):=\mathbb{E}\left[\prod_{e \in H \backslash\left\{e_{1}\right\}} \tilde{g}_{e}\left(x_{e}\right) \mid x_{J \backslash e_{1}} \in V_{J \backslash e_{1}}\right] .
\end{aligned}
$$

We have $g_{e_{1}}^{\prime} \leq \nu_{e_{1}}^{\prime}$ and $\tilde{g}_{e_{1}} \leq 1$ (pointwise). In the rest of this proof, unless otherwise specified, expectations are for functions on $V_{e_{1}}$ with arguments varying uniformly over $V_{e_{1}}$. The linear forms condition (4) implies that $\mathbb{E}\left[\nu_{e_{1}}^{\prime}\right]=1+o(1)$ and $\mathbb{E}\left[\left(\nu_{e_{1}}^{\prime}\right)^{2}\right]=$ $1+o(1)$, so that ${ }^{5}$

$$
\mathbb{E}\left[\left(\nu_{e_{1}}^{\prime}-1\right)^{2}\right]=o(1) .
$$

The square of the first term on the right-hand side of (30) equals

$$
\begin{aligned}
\mathbb{E}\left[g_{e_{1}}\left(g_{e_{1}}^{\prime}-\tilde{g}_{e_{1}}^{\prime}\right)\right]^{2} \leq \mathbb{E}\left[g_{e_{1}}\left(g_{e_{1}}^{\prime}-\tilde{g}_{e_{1}}^{\prime}\right)^{2}\right] \mathbb{E}\left[g_{e_{1}}\right] & \leq \mathbb{E}\left[\nu_{e_{1}}\left(g_{e_{1}}^{\prime}-\tilde{g}_{e_{1}}^{\prime}\right)^{2}\right] \mathbb{E}\left[\nu_{e_{1}}\right] \\
& =\left(\mathbb{E}\left[\left(g_{e_{1}}^{\prime}-\tilde{g}_{e_{1}}^{\prime}\right)^{2}\right]+o(1)\right)(1+o(1)) .
\end{aligned}
$$

The first inequality above is due to the Cauchy-Schwarz inequality. In the final step, both factors are estimated using Lemma 6.3 (for the first factor, expand the square $\left(g_{e_{1}}^{\prime}-\tilde{g}_{e_{1}}^{\prime}\right)^{2}$ and apply Lemma 6.3 term by term). Continuing (35) it suffices to show that the following quantity is $o_{\epsilon \rightarrow 0}(1)$ :

$$
\mathbb{E}\left[\left(g_{e_{1}}^{\prime}-\tilde{g}_{e_{1}}^{\prime}\right)^{2}\right]=\mathbb{E}\left[\left(g_{e_{1}}^{\prime}-\tilde{g}_{e_{1}}^{\prime}\right)\left(g_{e_{1}}^{\prime}-g_{e_{1}}^{\prime} \wedge 1\right)\right]+\mathbb{E}\left[\left(g_{e_{1}}^{\prime}-\tilde{g}_{e_{1}}^{\prime}\right)\left(g_{e_{1}}^{\prime} \wedge 1-\tilde{g}_{e_{1}}^{\prime}\right)\right]
$$

(here $a \wedge b:=\min \{a, b\}$ ). That is, we are capping the weighted hypergraph $g_{e_{1}}^{\prime}$ by 1. Since $\nu_{e_{1}}^{\prime}$ is very close to 1 by (34), this should not result in a large loss. Indeed, since $0 \leq g_{e_{1}}^{\prime} \leq \nu_{e_{1}}^{\prime}$, we have

$$
0 \leq g_{e_{1}}^{\prime}-g_{e_{1}}^{\prime} \wedge 1=\max \left\{g_{e_{1}}^{\prime}-1,0\right\} \leq \max \left\{\nu_{e_{1}}^{\prime}-1,0\right\} \leq\left|\nu_{e_{1}}^{\prime}-1\right| \text {. }
$$

Using (37), $g_{e_{1}}^{\prime} \leq \nu_{e_{1}}^{\prime}$, and $\tilde{g}_{e_{1}}^{\prime} \leq 1$, we bound the magnitude of the first term on the right-hand side of (36) by

$$
\begin{aligned}
& \mathbb{E}\left[\left(\nu_{e_{1}}^{\prime}+1\right)\left|\nu_{e_{1}}^{\prime}-1\right|\right]=\mathbb{E}\left[\left(\nu_{e_{1}}^{\prime}-1\right)\left|\nu_{e_{1}}^{\prime}-1\right|\right]+2 \mathbb{E}\left[\left|\nu_{e_{1}}^{\prime}-1\right|\right] \\
& \quad \leq \mathbb{E}\left[\left(\nu_{e_{1}}^{\prime}-1\right)^{2}\right]+2 \mathbb{E}\left[\left(\nu_{e_{1}}^{\prime}-1\right)^{2}\right]^{1 / 2}=o(1)
\end{aligned}
$$

\footnotetext{
${ }^{5}$ In fact, the only assumptions on $\nu$ needed for the proof of Theorem 2.17 are (34) and the strong linear forms condition, Lemma 6.3, as well as analogous conditions for other choices of $e_{1} \in H$ and allowing some subset of the functions $\nu_{e}$ to be replaced by 1 .
} 
by the triangle inequality, the Cauchy-Schwarz inequality, and (34). To estimate the second term on the right-hand side of (36), we need the following claim.

Claim. $\left(g_{e_{1}}^{\prime} \wedge 1, \tilde{g}_{e_{1}}^{\prime}\right)$ is an $\epsilon^{\prime}$-discrepancy pair with $\epsilon^{\prime}=o_{\epsilon \rightarrow 0}(1)$.

Proof of Claim. We need to show that, whenever $B_{f} \subseteq V_{f}$ for all $f \in \partial e_{1}$, we have

$$
\mathbb{E}\left[\left(g_{e_{1}}^{\prime}\left(x_{e_{1}}\right) \wedge 1-\tilde{g}_{e_{1}}^{\prime}\left(x_{e_{1}}\right)\right) \prod_{f \in \partial e_{1}} 1_{B_{f}}\left(x_{f}\right) \mid x_{e_{1}} \in V_{e_{1}}\right]=o_{\epsilon \rightarrow 0}(1) .
$$

Define $g_{e_{1}}^{\prime \prime}: V_{e_{1}} \rightarrow \mathbb{R}_{\geq 0}$ by $g_{e_{1}}^{\prime \prime}\left(x_{e_{1}}\right)=\prod_{f \in \partial e_{1}} 1_{B_{f}}\left(x_{f}\right)$. So the left-hand side of (38) is equal to

$$
\mathbb{E}\left[\left(g_{e_{1}}^{\prime} \wedge 1-g_{e_{1}}^{\prime}\right) g_{e_{1}}^{\prime \prime}\right]+\mathbb{E}\left[\left(g_{e_{1}}^{\prime}-\tilde{g}_{e_{1}}^{\prime}\right) g_{e_{1}}^{\prime \prime}\right]
$$

Using $0 \leq g_{e_{1}}^{\prime \prime} \leq 1$, (37), the Cauchy-Schwarz inequality, and (34), we can bound the magnitude of the first term in (39) by

$$
\mathbb{E}\left[\left|\nu_{e_{1}}^{\prime}-1\right|\right] \leq \mathbb{E}\left[\left(\nu_{e_{1}}^{\prime}-1\right)^{2}\right]^{1 / 2}=o(1) .
$$

The second term of (39) is equal to

$$
\mathbb{E}\left[\left(\prod_{e \in H \backslash\left\{e_{1}\right\}} g_{e}\left(x_{e}\right)-\prod_{e \in H \backslash\left\{e_{1}\right\}} \tilde{g}_{e}\left(x_{e}\right)\right) g_{e_{1}}^{\prime \prime}\left(x_{e_{1}}\right) \mid x \in V_{J}\right] .
$$

This is $o_{\epsilon \rightarrow 0}(1)$ by the induction hypothesis applied to new weighted hypergraphs where the old $\left(\nu_{e_{1}}, g_{e_{1}}, \tilde{g}_{e_{1}}\right)$ gets replaced by $\left(1, g_{e_{1}}^{\prime \prime}, g_{e_{1}}^{\prime \prime}\right)$, thereby decreasing $\mid\{e \in H$ : $\left.\nu_{e} \neq 1\right\} \mid$. Note that the linear forms condition continues to hold. Thus (38) holds, so $\left(g_{e_{1}}^{\prime} \wedge 1, \tilde{g}_{e_{1}}^{\prime}\right)$ is an $\epsilon^{\prime}$-discrepancy pair with $\epsilon^{\prime}=o_{\epsilon \rightarrow 0}(1)$.

We expand the second term of (36) as

$$
\mathbb{E}\left[\left(g_{e_{1}}^{\prime}-\tilde{g}_{e_{1}}^{\prime}\right)\left(g_{e_{1}}^{\prime} \wedge 1-\tilde{g}_{e_{1}}^{\prime}\right)\right]=\mathbb{E}\left[g_{e_{1}}^{\prime}\left(g_{e_{1}}^{\prime} \wedge 1\right)\right]-\mathbb{E}\left[g_{e_{1}}^{\prime} \tilde{g}_{e_{1}}^{\prime}\right]-\mathbb{E}\left[\tilde{g}_{e_{1}}^{\prime}\left(g_{e_{1}}^{\prime} \wedge 1\right)\right]+\mathbb{E}\left[\left(\tilde{g}_{e_{1}}^{\prime}\right)^{2}\right] .
$$

We claim that each expectation on the right-hand side of $(40)$ is $\mathbb{E}\left[\left(\tilde{g}_{e_{1}}^{\prime}\right)^{2}\right]+o_{\epsilon \rightarrow 0}(1)$. Indeed, by (32) and (33) we have

$$
\begin{aligned}
\mathbb{E} & {\left[g_{e_{1}}^{\prime}\left(g_{e_{1}}^{\prime} \wedge 1\right)\right]-\mathbb{E}\left[\left(\tilde{g}_{e_{1}}^{\prime}\right)^{2}\right] } \\
& =\mathbb{E}\left[\left(\left(g_{e_{1}}^{\prime}\left(x_{e_{1}}\right) \wedge 1\right) \prod_{e \in H \backslash\left\{e_{1}\right\}} g_{e}\left(x_{e}\right)-\tilde{g}_{e_{1}}^{\prime}\left(x_{e_{1}}\right) \prod_{e \in H \backslash\left\{e_{1}\right\}} \tilde{g}_{e}\left(x_{e}\right)\right) \mid x \in V_{J}\right],
\end{aligned}
$$

which is $o_{\epsilon \rightarrow 0}(1)$ by the induction hypothesis applied to new weighted hypergraphs where the old $\left(\nu_{e_{1}}, g_{e_{1}}, \tilde{g}_{e_{1}}\right)$ is replaced by $\left(1, g_{e_{1}}^{\prime} \wedge 1, \tilde{g}_{e_{1}}^{\prime}\right)$. This is allowed as $\left(g_{e_{1}}^{\prime} \wedge\right.$ $\left.1, \tilde{g}_{e_{1}}^{\prime}\right)$ is an $\epsilon^{\prime}$-discrepancy pair with $\epsilon^{\prime}=o_{\epsilon \rightarrow 0}(1)$, the new $\nu$ still satisfies the linear forms condition, and $\left|\left\{e \in H: \nu_{e} \neq 1\right\}\right|$ has decreased. The claims that the other terms on the right-hand side of $(40)$ are each $\mathbb{E}\left[\left(\tilde{g}_{e_{1}}^{\prime}\right)^{2}\right]+o_{\epsilon \rightarrow 0}(1)$ are similar (in fact, easier). It follows that (40) is $o_{\epsilon \rightarrow 0}(1)$, so (36) is $o_{\epsilon \rightarrow 0}(1)$ and we are done. 


\section{Concluding Remarks}

Conditions for counting lemmas. In this paper, we determined sufficient conditions for establishing a relative Szemerédi theorem and, more generally, a counting lemma for sparse hypergraphs. We have assumed that the hypergraph we want to count within is a subgraph of a pseudorandom hypergraph. The main question then is to determine a good notion of pseudorandomness which is suffficient to establish a counting lemma.

There is a marked difference between this paper and our previous paper on graphs [CFZ14] in terms of the type of pseudorandom condition assumed for the majorizing hypergraph. In this paper, we prove a counting lemma for a given hypergraph $H$ by assuming that the underlying pseudorandom hypergraph contains approximately the correct count for each hypergraph in a certain collection of hypergraphs $\mathcal{H}$ derived from $H$. That is, for each $H^{\prime} \in \mathcal{H}$, we assume that our pseudorandom hypergraph contains $(1+o(1)) p^{e\left(H^{\prime}\right)} n^{v\left(H^{\prime}\right)}$ labeled copies of $H^{\prime}$, where $p$ is the edge density of the pseudorandom hypergraph.

The approach used in [CFZ14] is equivalent, up to some polynomial loss in $\epsilon$, to assuming that the number of labeled cycles of length 4 in our pseudorandom graph is $(1+\epsilon) p^{4} n^{4}$, where $\epsilon$ is now a carefully controlled term and the question of whether $H$ can be embedded in our pseudorandom graph depends on whether $\epsilon$ is sufficiently small with respect to $H$ and $p$. It is possible to adapt the methods of this paper so that the notion of pseudorandomness used for hypergraphs is more closely related to this latter notion. However, for the purposes of applying the results to a relative Szemerédi theorem, the current formulation seemed more appropriate.

Gowers uniformity norms. For a function $f: \mathbb{Z}_{N} \rightarrow \mathbb{R}$, the Gowers $U^{r}$-norm of $f$ is defined to be

$$
\|f\|_{U^{r}}=\mathbb{E}\left[\prod_{\omega \in\{0,1\}^{r}} f\left(x_{0}+\omega \cdot \mathbf{x}\right) \mid x_{0}, x_{1}, \ldots, x_{r} \in \mathbb{Z}_{N}\right]^{1 / 2^{r}}
$$

where $\mathbf{x}=\left(x_{1}, \ldots, x_{r}\right)$. The following inequality, referred to as a generalized von Neumann theorem, bounds the weighted count of $(r+1)$-term arithmetic progressions from functions $f_{0}, \ldots, f_{r}$ in terms of the Gowers uniformity norm:

$$
\left|\mathbb{E}\left[f_{0}(x) f_{1}(x+d) f_{2}(x+2 d) \ldots f_{r}(x+r d) \mid x, d \in \mathbb{Z}_{N}\right]\right| \leq\left\|f_{j}\right\|_{U^{r}} \prod_{i \neq j}\left\|f_{i}\right\|_{\infty} .
$$

This fundamental fact is an important starting point for Gowers' celebrated proof [Gow01] of Szemerédi's theorem as well as many later developments in additive combinatorics. For a sparse set $S \subseteq \mathbb{Z}_{N}$ of density $p$, this inequality implies the correct count of $(r+1)$-term arithmetic progressions in $S$ as long as $\|\nu-1\|_{U^{r}}=$ $o\left(p^{r}\right)$, where $\nu=p^{-1} 1_{S}$ (a more careful analysis shows that it suffices to assume $\left.\|\nu-1\|_{U^{r}}=o\left(p^{r / 2}\right)\right)$. 
Gowers $[\text { Gow } 10]^{6}$ and Green [GreenPC] asked if $\|\nu-1\|_{U^{s}}=o(1)$ for some large $s=s(r)$ is sufficient for $\nu$ to satisfy a relative Szemerédi theorem for $(r+1)$ term arithmetic progressions. Note that this is precisely a linear forms condition and we proved in this paper that a different linear forms condition is sufficient. However, we do not even know if such a condition implies the existence of $(r+1)$ term arithmetic progressions in $\nu$. Clearly $s(r)$ cannot be too small and indeed we know from the recent work of Bennett and Bohman [BB] on the random AP-free process that one can find a 3 -AP-free $S \subset \mathbb{Z}_{N}$ such that $\nu=(N /|S|) 1_{S}$ satisfies $\|\nu-1\|_{U^{2}}=o(1)$. Therefore, if $s(2)$ exists, it must be greater than 2. More generally, they show that $s(r)>1+\log _{2} r$. In a companion note [CFZ], we show that if a measure $\nu$ satisfies the stronger condition $\|\nu-1\|_{U^{r}}=o\left(p^{r}\right)$, where $p=\|\nu\|_{\infty}^{-1}$, then the relative Szemerédi theorem holds with respect to $\nu$ for $(r+1)$-term arithmetic progressions. This strengthens the consequence of the generalized von Neumann theorem discussed above.

Corners in products of pseudorandom sets. Example 3.2 illustrates the relative multidimensional Szemerédi theorem applied to a pseudorandom set $S \subset \mathbb{Z}_{N}^{2}$. However, the situation is quite different for $S \times S \subset \mathbb{Z}_{N}^{2}$ with some pseudorandom set $S \subset \mathbb{Z}_{N}$. Indeed, $S \times S \subset \mathbb{Z}_{N}^{2}$ does not satisfy the linear forms condition in Example 3.2. Intuitively, this is because the events $(x, y) \in S \times S$ and $\left(x, y^{\prime}\right) \in S \times S$ are correlated as both involve $x \in S$.

However, we may still deduce the following result using our relative triangle removal lemma. Recall that a corner in $\mathbb{Z}_{N}^{2}$ is a set of the form $\{(x, y),(x+d, y),(x, y+$ $d)\}$, where $d \neq 0$.

Proposition 7.1. If $S \subset \mathbb{Z}_{N}$ is such that $\nu=\frac{N}{|S|} 1_{S}$ satisfies

$$
\begin{aligned}
& \mathbb{E}\left[\nu(x) \nu\left(x^{\prime}\right) \nu(z-x) \nu\left(z-x^{\prime}\right) \nu\left(z^{\prime}-x\right) \nu\left(z^{\prime}-x^{\prime}\right)\right. \\
& \left.\cdot \nu(y) \nu\left(y^{\prime}\right) \nu(z-y) \nu\left(z-y^{\prime}\right) \nu\left(z^{\prime}-y\right) \nu\left(z^{\prime}-y^{\prime}\right) \mid x, x^{\prime}, y, y^{\prime}, z, z^{\prime} \in \mathbb{Z}_{N}\right]=1+o(1)
\end{aligned}
$$

and similar conditions hold if any subset of the $\nu$ factors are erased, then any cornerfree subset of $S \times S$ has size $o\left(|S|^{2}\right)$.

Proof (sketch). Let $A$ be a corner-free subset of $S \times S$. We build two tripartite graphs $\Gamma$ and $G$ on the same vertex set $X \cup Y \cup Z$ with $X=Y=S$ and $Z=\mathbb{Z}_{N}$ (note that unlike the proof of Theorem 3.1 we do not take $X$ and $Y$ to be the whole of $\mathbb{Z}_{N}$ here). In $\Gamma$, we place a complete bipartite graph between $X$ and $Y$; between $Y$ and $Z$ the edge $(y, z) \in Y \times Z$ is present if and only if $z-y \in S$; and between $X$ and $Z$ the edge $(x, z) \in X \times Z$ is present if and only if $z-x \in S$. In $G$, between $X$ and $Y$ the edge $(x, y) \in(X, Y)$ is present if and only if $(x, y) \in A$; between $Y$ and $Z$ the edge $(y, z) \in Y \times Z$ is present if and only if $(z-y, y) \in A$; and between $X$ and $Z$ the edge $(x, z) \in X \times Z$ is present if and only if $(x, z-x) \in A$.

\footnotetext{
${ }_{6}$ This question can be found in the penultimate paragraph in $\S 4$ of the arXiv version of [Gow10].
} 
The vertices $(x, y, z) \in X \times Y \times Z$ form a triangle if and only if $(x, y),(z-$ $y, y),(x, z-x) \in A$. These three points form a corner, which is degenerate only when $x+y=z$. Since $A$ is corner-free, every edge of $G$ is contained in exactly one triangle (namely the one that completes the equation $x+y=z$ ). In particular, $G$ contains exactly $|A|$ triangles. After checking some hypotheses, we can apply our relative triangle removal lemma (as a special case of Theorem 2.12) to conclude that it is possible to remove all triangles from $G$ by deleting $o\left(|S|^{2}\right)$ edges. Since every edge of $G$ is contained in exactly one triangle, and $|G|$ has $3|A|$ edges, we have $|A|=o\left(|S|^{2}\right)$, as desired.

One can easily generalize the above Proposition to $S^{m} \subset \mathbb{Z}_{N}^{m}$ (as before, $S \subset$ $\left.\mathbb{Z}_{N}\right)$. Here a corner is a set of the form $\left\{\mathbf{x}, \mathbf{x}+d \mathbf{e}_{1}, \ldots, \mathbf{x}+d \mathbf{e}_{m}\right\}$, where $\mathbf{x} \in \mathbb{Z}_{N}$, $0 \neq d \in \mathbb{Z}_{N}$, and $\mathbf{e}_{i}$ is the $i$-th coordinate vector. Then, for any fixed $m$, any cornerfree subset of $S^{m}$ must have size $o\left(|S|^{m}\right)$, provided that $\nu=\frac{N}{|S|} 1_{S}$ satisfies the linear forms condition

$$
\begin{aligned}
\mathbb{E} & {\left[\prod_{i=1}^{m}\left(\nu\left(x_{i}^{(0)}\right)^{n_{i, 0}} \nu\left(x_{i}^{(1)}\right)^{n_{i, 1}} \prod_{\omega \in\{0,1\}\{0\} \cup[m] \backslash\{i\}}\left(x_{0}^{\left(\omega_{0}\right)}-\sum_{j \in[m] \backslash\{i\}} x_{j}^{\left(\omega_{j}\right)}\right)^{n_{i, \omega}}\right)\right.} \\
& \left.\mid x_{0}^{(0)}, x_{0}^{(1)}, \ldots, x_{m}^{(0)}, x_{m}^{(1)} \in \mathbb{Z}_{N}\right]=1+o(1)
\end{aligned}
$$

for any choices of exponents $n_{i, 0}, n_{i, 1}, n_{i, \omega} \in\{0,1\}$.

A more general result concerning the existence of arbitrarily shaped constellations in $S^{m}$ is known, provided that $S$ satisfies certain stronger linear forms hypotheses. We refer the readers to [CMT,FZ, TZ13] for further details. In particular, the multidimensional relative Szemerédi theorem holds in $P^{m}$, where $P$ is the primes.

Sparse graph limits. The regularity method played a fundamental role in the development of the theory of dense graph limits [BCLSV08, LS06]. However, no satisfactory theory of graph limits is known for graphs with edge density $o(1)$. Bollobás and Riordan [BR09] asked a number of questions and made explicit conjectures on suitable conditions for sparse graph limits and counting lemmas. Our work gives some natural sufficient conditions for obtaining a counting lemma in a sequence of sparse graphs $G_{N}$. The new counting lemma allows us to transfer the results of Lovász and Szegedy [LS06,LS07] on the existence of the limit graphon, as well as the results of Borgs, Chayes, Lovász, Sós, and Vesztergombi [BCLSV08] on the equivalence of left-convergence (i.e., convergence in homomorphism densities) and convergence in cut distance. The famous quasirandomness results of Chung, Graham, and Wilson [CGW89] also transfer, namely, that an appropriate relationship between edge density and $C_{4}$-density (of homomorphisms) determines the asymptotic $F$-density for every graph $F$. We will explain these connections in more detail in future writing.

Existing applications of the Green-Tao method. Though our discussion has focused on the relative Szemerédi theorem, we have proved a relative version of 
the stronger multidimensional Szemerédi theorem. Following Tao [Tao06a], this may be used to prove that the Gaussian primes contain arbitrarily shaped constellations, though without the need to verify either the correlation condition or the dual function condition. It seems likely that our method could also be useful for simplifying several other papers where the machinery of Green and Tao is used [CM12, GT10,Le11, Mat12a, Mat12b, TZ08]. In some cases it should be possible to use our results verbatim but in others, such as the paper of Tao and Ziegler [TZ08] proving that there are arbitrarily long polynomial progressions in the primes, it will probably require substantial additional work.

Sparse hypergraph regularity. In proving a hypergraph removal lemma for subgraphs of pseudorandom hypergraphs, we have developed a general approach to regularity and counting in sparse pseudorandom hypergraphs which has the potential for much broader application. It is, for example, quite easy to use our results to prove analogues of well-known combinatorial theorems such as Ramsey's theorem and Turán's theorem relative to sparse pseudorandom hypergraphs of density $N^{-c_{H}}$. We omit the details. In the graph case, a number of further applications were discussed in [CFZ14]. We expect that hypergraph versions of many of these applications should be an easy corollary of our results.

Counting in random hypergraphs. There has been much recent work on counting lemmas and relative versions of combinatorial theorems within random graphs and hypergraphs [BMS, CG, CGSS14,ST,Sch]. Surprisingly, there are a number of disparate approaches to these problems, each having its own strengths and weaknesses. We believe that our results can be used to give an alternative framework for one of these approaches, due to Conlon and Gowers [CG]. ${ }^{7}$ Their proof relies heavily upon an application of the Green-Tao transference theorem, which we believe can be replaced with an application of the sparse Frieze-Kannan regularity lemma and our densification technique. However, the key technical step in [CG], which in our language is to verify that the strong linear forms condition, Lemma 6.3, holds when $\nu$ is a random measure, would remain unchanged.

Sparse arithmetic removal. In Theorem 3.3, we proved an arithmetic removal lemma for linear patterns such as arithmetic progressions. More generally, an arithmetic removal lemma claims that if a system of linear equations $M a=b$ over the integers has a small number of solutions $a=\left(a_{1}, a_{2}, \ldots, a_{n}\right)$ with $a_{i} \in A_{i}$ for all $i=1,2, \ldots, n$ then one may remove a small number of elements from each $A_{i}$ to find subsets $A_{i}^{\prime}$ such that there are no solutions $a^{\prime}=\left(a_{1}^{\prime}, a_{2}^{\prime}, \ldots, a_{n}^{\prime}\right)$ to $M a^{\prime}=b$ with $a_{i}^{\prime} \in A_{i}^{\prime}$ for all $i=1,2, \ldots, n$. Such a result was conjectured by Green [Gre05] and proved by Král', Serra, and Vena [KSV12] and, independently, Shapira [Sha10]. Both of these proofs are based upon representing a system of linear equations by a hypergraph and deducing the arithmetic removal lemma from a hypergraph removal

7 This should at least be true for theorems regarding graphs and hypergraphs, though we feel that a similar approach should also be possible for subsets of the integers. 
lemma. Such an idea was first used by Král', Serra, and Vena [KSV09] with graphs (instead of hypergraphs). In [CFZ14], we adapted the arguments of [KSV09] to sparse pseudorandom subsets of the integers using the removal lemma in sparse pseudorandom graphs. Likewise, our results on hypergraph removal in this paper may be used to prove a sparse pseudorandom generalization of the arithmetic removal lemma [KSV12, Sha10] for all systems of linear equations.

\section{Acknowledgments}

We would like to thank Tom Bohman and Ben Green for helpful discussions.

\section{References}

[BMS] J. Balogh, R. Morris, and W. SAmotiJ. Independent sets in hypergraphs. J. Amer. Math. Soc., to appear.

[BB] P. Bennett and T. Bohman. A note on the random greedy independent set algorithm. arXiv:1308.3732.

[BR09] B. Bollobás and O. Riordan. Metrics for sparse graphs. In: Surveys in combinatorics 2009, London Math. Soc. Lecture Note Ser., vol. 365, Cambridge University Press, Cambridge, 2009, pp. 211-287.

[BClSV08] C. Borgs, J.T. Chayes, L. Lovász, V.T. Sós, and K. Vesztergombi. Convergent sequences of dense graphs. I. Subgraph frequencies, metric properties and testing. Adv. Math., 219 (2008), 1801-1851.

[CGW89] F.R.K. Chung, R.L. Graham, and R.M. Wilson. Quasi-random graphs. Combinatorica, 9 (1989), 345-362.

[CCF09] A. Coja-Oghlan, C. Cooper, and A. Frieze. An efficient sparse regularity concept. SIAM J. Discrete Math., 23 (2009/10), 2000-2034.

[CFZ14] D. Conlon, J. Fox, and Y. Zhao. Extremal results in sparse pseudorandom graphs. Adv. Math., 256 (2014), 206-290.

[CFZ] D. Conlon, J. Fox, and Y. Zhao. Linear forms from the Gowers uniformity norm. Unpublished companion note.

[CG] D. Conlon and W.T. Gowers, Combinatorial theorems in sparse random sets. arXiv:1011.4310.

[CGSS14] D. Conlon, W.T. Gowers, W. SamotiJ, and M. Schacht. On the KŁR conjecture in random graphs. Israel J. Math., 203 (2014), 535-580.

[CM12] B. Cook and A. Magyar. Constellations in $\mathbb{P}^{d}$. Int. Math. Res. Not., 2012 (2012), 2794-2816.

[CMT] B. Cook, A. Magyar, and T. Titichetrakun. A multidimensional Szemerédi theorem in the primes. arXiv:1306.3025.

[FZ] J. Fox and Y. ZhaO. A short proof of the multidimensional Szemerédi theorem in the primes. Amer. J. Math., to appear.

[FR02] P. FRANKL and V. RÖDL. Extremal problems on set systems. Random Structures Algorithms, 20 (2002), 131-164.

[FK99] A. FRIEZE and R. KANNAN. Quick approximation to matrices and applications. Combinatorica, 19 (1999), 175-220.

[FK78] H. Furstenberg and Y. Katznelson. An ergodic Szemerédi theorem for commuting transformations. J. Analyse Math., 34 (1978), 275-291. 
[GY03] D.A. Goldston and C.Y. Yildirim. Higher correlations of divisor sums related to primes. I. Triple correlations. Integers, 3 (2003), A5, 66.

[Gow01] W.T. Gowers. A new proof of Szemerédi's theorem. Geom. Funct. Anal. 11 (2001), 465-588.

[Gow07] W.T. Gowers. Hypergraph regularity and the multidimensional Szemerédi theorem. Ann. of Math., 166 (2007), 897-946.

[Gow10] W.T. Gowers. Decompositions, approximate structure, transference, and the Hahn-Banach theorem. Bull. Lond. Math. Soc., 42 (2010), 573-606. arXiv:0811.3103.

[GreenPC] B. Green. Personal communication.

[Gre05] B. GREen. A Szemerédi-type regularity lemma in abelian groups, with applications. Geom. Funct. Anal., 15 (2005), 340-376.

[GT08] B. Green and T. TAO. The primes contain arbitrarily long arithmetic progressions. Ann. of Math., 167 (2008), 481-547.

[GT10] B. Green and T. TaO. Linear equations in primes. Ann. of Math., 171 (2010), $1753-1850$.

[Koh97] Y. Kohayakawa. Szemerédi's regularity lemma for sparse graphs. Foundations of computational mathematics (Rio de Janeiro, 1997), Springer, Berlin, 1997, pp. 216-230.

[KSV09] D. KrÁL', O. Serra, and L. Vena. A combinatorial proof of the removal lemma for groups. J. Combin. Theory Ser. A, 116 (2009), 971-978.

[KSV12] D. KrÁL', O. Serra, and L. Vena. A removal lemma for systems of linear equations over finite fields. Israel J. Math. 187 (2012), 193-207.

[Le11] T.H. LÊ. Green-Tao theorem in function fields. Acta Arith. 147 (2011), 129152.

[LS06] L. Lovász and B. Szegedy. Limits of dense graph sequences. J. Combin. Theory Ser. B 96 (2006), 933-957.

[LS07] L. LovÁsz and B. Szegedy. Szemerédi's lemma for the analyst. Geom. Funct. Anal. 17 (2007), 252-270.

[Mat12a] L. Matthiesen, Correlations of the divisor function. Proc. Lond. Math. Soc. 104 (2012), 827-858.

[Mat12b] L. MATthiESEn. Linear correlations amongst numbers represented by positive definite binary quadratic forms. Acta Arith. 154 (2012), 235-306.

[NRS06] B. Nagle, V. RödL, and M. Schacht. The counting lemma for regular $k$ uniform hypergraphs. Random Structures Algorithms 28 (2006), 113-179.

[RTTV08] O. Reingold, L. Trevisan, M. Tulsiani, and S. Vadhan. Dense Subsets of Pseudorandom Sets. In: 49th Annual IEEE symposium on foundations of computer science, IEEE Computer Society (2008), pp. 76-85.

[RS04] V. RÖDL and J. SkOKAN. Regularity lemma for $k$-uniform hypergraphs. Random Structures Algorithms 25 (2004), 1-42.

[RS06] V. RöDL and J. SkokAN. Applications of the regularity lemma for uniform hypergraphs. Random Structures Algorithms. 28 (2006), 180-194.

[RS78] I.Z. RuZSA and E. SzemeRÉDI. Triple systems with no six points carrying three triangles. In: Combinatorics (Proc. Fifth Hungarian Colloq., Keszthely, 1976), Vol. II, Colloq. Math. Soc. János Bolyai, vol. 18, North-Holland, Amsterdam (1978), pp. 939-945.

[ST] D. Saxton and A. Thomason. Hypergraph containers. Invent. Math., to appear. 
[Sch] M. Schacht. Extremal results for random discrete structures. Submitted.

[Sco11] A. Scotr. Szemerédi's regularity lemma for matrices and sparse graphs. Combin. Probab. Comput. 20 (2011), 455-466.

[Sha10] A. ShAPIRA. A proof of Green's conjecture regarding the removal properties of sets of linear equations. J. Lond. Math. Soc. 81 (2010), 355-373.

[Sol03] J. Solymosi. Note on a Generalization of Roth's Theorem. In: Discrete and computational geometry, Algorithms Combin., vol. 25, Springer, Berlin (2003), pp. 825-827.

[Sol04] J. Solymosi. A note on a question of Erdős and Graham. Combin. Probab. Comput. 13 (2004), 263-267.

[Sze75] E. SzEmERÉDI. On sets of integers containing no $k$ elements in arithmetic progression. Acta Arith. 27 (1975), 199-245.

[Tao] T. TAO. A remark on Goldston-Yıldırım correlation estimates. Unpublished.

[Tao06a] T. TAO. The Gaussian primes contain arbitrarily shaped constellations. J. Anal. Math. 99 (2006), 109-176.

[Tao06b] T. TAO. A variant of the hypergraph removal lemma. J. Combin. Theory Ser. A 113 (2006), 1257-1280.

[TZ08] T. TAO and T. ZIEGLER. The primes contain arbitrarily long polynomial progressions. Acta Math., 201 (2008), 213-305.

[TZ13] T. TAO and T. Ziegler. A multi-dimensional Szemerédi theorem for the primes via a correspondence principle. Israel J. Math., to appear.

[Tow] H. Towsner. An analytic approach to sparse hypergraphs: hypergraph removal. arXiv:1204.1884.

[TTV09] L. Trevisan, M. Tulsiani, and S. Vadhan. Regularity, Boosting, and Efficiently Simulating Every High-entropy Distribution. In: 24th Annual IEEE Conference on Computational Complexity, IEEE Computer Society, 2009, pp. 126136.

[Zh14] Y. Zhaо. An arithmetic transference proof of a relative Szemerédi theorem. Math. Proc. Cambridge Philos. Soc. 156 (2014), 255-261.

DAVID CONLON

Mathematical Institute, Oxford OX1 3LB, UK

david.conlon@maths.ox.ac.uk

JACOB Fox and Yufei ZHAO

Department of Mathematics, MIT, Cambridge, MA 02139-4307, USA

$$
\begin{array}{r}
\text { fox@math.mit.edu } \\
\text { yufeiz@math.mit.edu }
\end{array}
$$

Received: September 10, 2014

Accepted: October 20, 2014 